\title{
Stellar evolution with rotation
}

\section{Wolf-Rayet star populations at different metallicities}

\author{
G. Meynet and A. Maeder
}

Geneva Observatory, 1290 Sauverny, Switzerland

e-mail: [Georges.Meynet;Andre.Maeder]@obs.unige.ch

Received 20 January 2004 / Accepted 28 June 2004

\begin{abstract}
Grids of models of massive stars $\left(M \geq 20 M_{\odot}\right)$ with rotation are computed for metallicities $Z$ ranging from that of the Small Magellanic Cloud (SMC) to that of the Galactic Centre. The hydrostatic effects of rotation, the rotational mixing and the enhancements of the mass loss rates by rotation are included. The evolution of the surface rotational velocities of the most massive O-stars mainly depends on the mass loss rates and thus on the initial $Z$ value. The minimum initial mass for a star for entering the Wolf-Rayet (WR) phase is lowered by rotation. For all metallicities, rotating stars enter the WR phase at an earlier stage of evolution and the WR lifetimes are increased, mainly as a result of the increased duration of the eWNL phase. Models of WR stars predict in general rather low rotation velocities $\left(<50 \mathrm{~km} \mathrm{~s}^{-1}\right)$ with a few possible exceptions, particularly at metallicities lower than solar where WR star models have in general faster rotation and more chance to reach the break-up limit. The properties of the WR populations as predicted by the rotating models are in general in much better agreement with the observations in nearby galaxies. Some possible remaining difficulties in these comparisons are mentioned. The evolution of the chemical abundances is largely influenced by rotation in all phases from the MS phase to the WN and WC phases. We also show that the interval of initial masses going through the LBV stage is changing with $Z$ and $\Omega$.

The observed variation with metallicity of the fractions of type Ib/Ic supernovae with respect to type II supernovae as found by Prantzos \& Boissier (2003) is very well reproduced by the rotating models, while non-rotating models predict much too low ratios. This indicates that the minimum initial masses of single stars going through a WR phase are consistently predicted. At $Z=0.040$, stars with initial masses above $50 M_{\odot}$ reach a final mass at the time of supernova explosion between 5 and $7.5 M_{\odot}$, while at $Z=0.004$, like in the SMC, the final masses of stars are in the range of 17-29 $M_{\odot}$. On the whole, rotation appears to be an essential parameter even for the WR properties. Detailed tables describing the evolutionary tracks are available on the web.
\end{abstract}

Key words. stars: evolution - stars: rotation - stars: Wolf-Rayet

\section{Introduction}

Wolf-Rayet stars are considered to be bare stellar cores whose original H-rich envelopes have been removed either by strong stellar winds or by mass transfer through Roche Lobe Overflow in close binary systems (Conti 1976; Chiosi \& Maeder 1986; Abbott \& Conti 1987). Their associations with young star forming regions implies that their progenitors must be massive stars (see e.g. the recent review by Massey 2003, and references therein). WR stars have a deep impact on their surroundings thanks to their high luminosity and their strong stellar winds. Their broad emission lines can be detected in the integrated spectrum of remote galaxies (Kunth \& Sargent 1981; Schaerer et al. 1999) enabling us to study star formation and evolution in very different environments, from metal poor blue compact dwarf galaxies to the vicinity of AGN (see e.g. Lípari et al. 2003). They contribute to the enrichment of the interstellar medium by newly synthesized elements. In particular their winds at high metallicity may be heavily loaded with carbon (Maeder 1992). Their winds may also eject significant amounts of ${ }^{26} \mathrm{Al}$ (see e.g. Vuissoz et al. 2004), responsible for the diffuse emission at $1.8 \mathrm{MeV}$ observed in the plane of our Galaxy (Prantzos \& Diehl 1996), in ${ }^{19} \mathrm{~F}$ (Meynet \& Arnould 2000) whose origin still remains largely unknown (Cunha et al. 2003), and in s-process elements (see e.g. Arnould et al. 1997). The WC star winds are also rich in ${ }^{22} \mathrm{Ne}$, which explains the high ${ }^{22} \mathrm{Ne} /{ }^{20} \mathrm{Ne}$ isotopic ratio observed in the galactic cosmic ray source material (see e.g. Meynet et al. 2001). WR stars are also the progenitors of type Ib/Ic supernovae (see the review by Hamuy 2003). Recently the spectrum of such a supernova was observed in the optical transient of a $\gamma$-ray burst (see e.g. Hjorth et al. 2003), confirming the suspected link between these stars and the long $\gamma$-ray bursts (Woosley 1993). For all these reasons Wolf-Rayet stars appear as objects worthwhile to be well understood.

In a previous paper (Meynet \& Maeder 2003, Paper X), we discussed the consequences of rotation on the properties of WR stars at solar metallicity. One of the main conclusions is that the theoretical predictions for the number ratios of Wolf-Rayet to O-type stars, for the ratio of WN to WC stars and 
for the fraction of WR stars in the transition WN/WC phase, are in good agreement with the observations when the effects of rotation are accounted for in stellar models. In contrast, the models with present-day mass loss rates and no rotation do not succeed in reproducing the observed values. The main purpose of the present paper is to explore the case of metallicities lower and higher than solar and to see if the above conclusions still hold.

Let us recall that interesting questions arise from the observations of WR stars both at low and high metallicity. At low metallicity, it has generally been thought that WR stars might preferentially be formed by mass transfer through Roche Lobe Overflow in close binary systems. For instance, it was thought that the majority, if not all the WR stars in the SMC should be born thanks to the mass transfer mechanism. The main reason is that, at low metallicity, the mass loss rates are much lower than at higher metallicity, thus making the ejection of the H-rich envelope by stellar winds more difficult. However, this idea was recently challenged by the works of Foellmi et al. (2003a,b). They looked for periodic radial velocity variability in all the WR stars in the Small Magellanic Cloud and in two thirds of the WR stars in the Large Magellanic Cloud. They found that the percentage of binaries among the WR stars is of the order of $40 \%$ for the SMC and $30 \%$ for the LMC, thus comparable or even below the percentage of binaries among the WR stars in our Galaxy. This means that, in the SMC, at most $40 \%$ of the WR stars could originate from mass transfer through Roche Lobe Overflow (RLOF) in a close binary system. The real fraction is likely lower since RLOF has not necessarily occurred in all these systems. Therefore even in the SMC, a large fraction of the WR stars likely originate via the single star scenario.

How is it possible? Does this mean that the mass loss rates are larger than usually found or that another process is at work which favours the evolution of massive stars into the WR phase? In an earlier work (Maeder \& Meynet 1994) we explored the first hypothesis, namely the effects of enhanced mass loss rates. We multiplied by a factor of two the mass loss rates given by de Jager et al. (1988) during the O-type star phase and we adopted an average mass loss rate of $8 \times 10^{-5} M_{\odot} \mathrm{yr}^{-1}$ during the WNL phase, i.e. twice the average mass loss rate given by Abbott \& Conti (1987). Doing so, we obtained a good agreement with the observed WR populations for the metallicities between that of the SMC and up to twice the solar metallicity. However, nowadays clear evidence of clumping in stellar winds of massive stars (Nugis et al. 1998) has been found and the new estimates of the mass loss rates (Nugis \& Lamers 2000) are reduced by a factor 2 to 3 with respect to the enhanced mass loss rates described above. Therefore the enhanced mass loss rate hypothesis is ruled out and another process must be at work. In Paper X, we showed that rotation might well be this process. Indeed, in massive star models, rotation favours the evolution into the WR phase in two ways, first by allowing the star to enter into the WR phase at an earlier stage, thus making the WR lifetime longer and secondly by allowing smaller initial mass stars to go through a WR phase. These effects, as we shall see below, are also present at low metallicity and allow us to reproduce the observed WR populations in the LMC and
SMC without assuming that a large fraction of WR stars owe their existence to mass transfer in close binary systems.

We shall also study the effects of rotation at higher metallicity than solar, more precisely at twice the solar metallicity, a value often quoted for the galactic centre (although some authors quote for this region a value of the metallicity similar to that of the solar neighbourhood, see Carr et al. 1999; Najarro 2003). This region of the Milky Way is very rich in massive stars (according to Figer et al. 2002, the Arches cluster contains about $5 \%$ of all known WR stars in the Galaxy) and it is thus interesting to derive the properties of the WR stars predicted by the present rotating models. Moreover, populations of Wolf-Rayet stars at a higher metallicity may also be of interest for studying the stellar population in the vicinity of AGNs (see e.g. the review by Heckman 1999).

Section 2 briefly summarizes the physics of the models. Evolution of the surface rotational velocities is discussed in Sect. 3. The evolutionary tracks are presented in Sect. 4 . The effects of rotation on WR star formation and WR lifetimes at different metallicities are discussed in Sect. 5. Comparisons with the observed WR populations are performed in Sect. 6. Finally the predicted surface abundances of the present WR stellar models are discussed in Sect. 7.

\section{Physics of the models}

Most of the physical ingredients of the present models are the same as in the solar metallicity models of Meynet \& Maeder (2003, Paper X). They differ in only two points:

- The initial compositions are adapted for the different metallicities considered here. For a given metallicity $Z$ (in mass fraction), the initial helium mass fraction $Y$ is given by the relation $Y=Y_{\mathrm{p}}+\Delta Y / \Delta Z \cdot Z$, where $Y_{\mathrm{p}}$ is the primordial helium abundance and $\Delta Y / \Delta Z$ the slope of the helium-to-metal enrichment law. We use the same values as in Maeder \& Meynet (2001) i.e. $Y_{\mathrm{p}}=0.23$ and $\Delta Y / \Delta Z=$ 2.5. For the metallicities $Z=0.004,0.008$ and 0.040 considered in this work, we thus have $X=0.757,0.744,0.640$ and $Y=0.239,0.248,0.320$ respectively. For the heavy elements we adopt the same mixture as the one used to compute the opacity tables for solar composition. In that respect the present grid differs also from our previous non-solar metallicity rotating models (Papers VII and VIII). In these models, we adopted the "enhanced alpha elements" opacity tables of Iglesias \& Rogers (1996) instead of the "solar composition" ones. As the relative abundances of $\alpha$-nuclei vary according to the galactic history, the present choice may be better.

- Secondly the wind anisotropies induced by rotation were neglected. This last choice appears justified in view of the results obtained in Paper X. Indeed for the initial velocities considered $\left(v_{\text {ini }}=300 \mathrm{~km} \mathrm{~s}^{-1}\right)$, the effects of the wind anisotropies have been shown to be very small. Let us however emphasize that this is not true for higher initial velocities (Maeder 2002).

Since mass loss rates are a key ingredient of the models in the mass range considered here, let us recall the prescriptions used. 
The changes of the mass loss rates $\dot{M}$ with rotation are taken into account as explained in Maeder \& Meynet (2000a). As reference mass loss rates we adopt the mass loss rates of Vink et al. $(2000,2001)$ who take account of the occurrence of bistability limits which change the wind properties and mass loss rates. For the domain not covered by these authors we use the empirical law devised by de Jager et al. (1988). Note that this empirical law, which presents a discontinuity in the mass flux near the Humphreys-Davidson limit, implicitly accounts for the mass loss rates of LBV stars. For the non-rotating models, since the empirical values for the mass loss rates are based on stars covering the whole range of rotational velocities, we must apply a reduction factor to the empirical rates to make them correspond to the non-rotating case. The same reduction factor was used as in Maeder \& Meynet (2001). During the Wolf-Rayet phase we use the mass loss rates by Nugis \& Lamers (2000). These mass loss rates, which account for the clumping effects in the winds, are smaller by a factor 2-3 than the mass loss rates used in our previous non-rotating "enhanced mass loss rate" stellar grids (Meynet et al. 1994).

During the non-WR phases of the present models, we assumed that the mass loss rates depend on the initial metallicity as $\dot{M}(Z)=\left(Z / Z_{\odot}\right)^{1 / 2} \dot{M}\left(Z_{\odot}\right)$ (Kudritzki \& Puls 2000; Vink et al. 2001). For models at $Z=0.040$, we also compute a series of models with metallicity dependent mass loss rates during the WR phase. According to Crowther et al. (2002) mass loss rates during the WR phase may show the same metallicity dependence as the winds of O-type stars, i.e. scale with $\sim\left(Z / Z_{\odot}\right)^{1 / 2}$.

The effective temperature of Wolf-Rayet stars is a delicate problem, since the winds may have a non-negligible optical thickness. Here we adopt a simple correction scheme to take account of this effect (e.g. Langer 1989): the effective radius $R_{\text {eff }}$ at the optical thickness $\tau=2 / 3$ is related to the classical photospheric radius $R$ by the relation

$R_{\mathrm{eff}}=R+\frac{3 K|\dot{M}|}{8 \pi v_{\infty}}$

where $K$ is the opacity and the other symbols have their usual meaning. More details on the procedure to estimate $K, \tau$ and $v_{\infty}$ are given in Schaller et al. (1992). The effective temperature at $\tau=2 / 3$ is then obtained by the usual relation $L=4 \pi R_{\text {eff }}^{2} \sigma T_{\text {eff }}^{4}$. Such a correction has been applied in the WR stages and only there.

A moderate overshooting is included in the present rotating and non-rotating models. The radius of the convective cores are increased with respect to their values obtained by the Schwarzschild criterion by a quantity equal to $0.1 H_{\mathrm{p}}$, where $H_{\mathrm{p}}$ is the pressure scale height estimated at the Schwarzschild boundary. The effect of rotation on the transport of the chemical species and of the angular momentum are included as in our Papers VII and VIII.

As initial rotation, we have considered a value equal to $300 \mathrm{~km} \mathrm{~s}^{-1}$ on the ZAMS for all the initial masses and metallicities considered. At solar metallicity, this initial value produces time-averaged equatorial velocities on the MS well in the observed range, i.e. between 200 and $250 \mathrm{~km} \mathrm{~s}^{-1}$. At low metallicities this initial rotational velocity corresponds also to mean values between 200 and $250 \mathrm{~km} \mathrm{~s}^{-1}$ on the MS phase, while at twice the solar metallicity, the mean velocity is lower, between 160 and $230 \mathrm{~km} \mathrm{~s}^{-1}$ (see Table 1). Presently we do not know the distribution of the rotational velocities at these non-solar metallicities and thus we do not know if the adopted initial velocity corresponds to the average observed values. It may be that at lower metallicities the initial velocity distribution contains a larger number of high initial velocities (Maeder et al. 1999), in which case the effects of rotation described below would be underestimated at low metallicity.

All the models were computed up to the end of the helium-burning phase. Their further evolution in the advanced stages will be presented in a forthcoming paper (Hirschi et al. in press). In order to facilitate future detailed comparisons, we provide electronic tables describing the evolutionary tracks presented in this paper ${ }^{1}$. For each initial mass, we extracted 350 points describing the whole sequence from the ZAMS to the end of the core He-burning phase. At each time step we provide the following quantities: age in years, actual mass in solar masses, $\log L / L_{\odot}, \log T_{\text {eff }}$ (which takes account of the optical thickness of the winds for WR stars, see above), the surface abundances in mass fraction of $\mathrm{H}, \mathrm{He},{ }^{12} \mathrm{C},{ }^{13} \mathrm{C},{ }^{14} \mathrm{~N},{ }^{16} \mathrm{O},{ }^{17} \mathrm{O}$, ${ }^{18} \mathrm{O},{ }^{20} \mathrm{Ne},{ }^{22} \mathrm{Ne}$, the fraction of the mass of the star occupied by the convective core, the uncorrected effective temperature, i.e. which does not take account of the optical thickness of the winds (for WR stars only), the mass loss rate in solar masses per year, $\log \rho_{\mathrm{c}}$, the central density, $\log T_{\mathrm{c}}$, the central temperature, the abundances at the centre of the star in mass fraction of the same elements as above, the ratio of the polar radius to the equatorial radius, the surface equatorial velocity in $\mathrm{km} \mathrm{s}^{-1}$, and the ratio of the surface angular velocity to the break-up velocity. For the nuclear burning phases, models with the same number in the tables always correspond to the same evolutionary stage, i.e. have the same mass fraction of hydrogen or helium at the centre: point 1 corresponds to the ZAMS stage, point 100 to the end of the core H-burning phase, point 201 and 350 to the beginning and end respectively of the core He-burning stage.

To conclude this section, let us emphasize that the amplitudes of the effects studied in this paper are particularly sensitive to the mass loss rates, the extension of the convective cores and the initial rotation. Does this mean that the present results are very model-dependent? We do not think so for the following reasons: firstly we are not free to change the above parameters beyond certain limits, for instance the initial rotation was chosen in order to reproduce the averaged observed velocity in the MS band, mass loss comes in part from the radiation wind theory, in part from empirically based relations, the overshoot is constrained from comparisons between the observed and computed MS width; secondly the present physical ingredients already allowed us to reproduce many observed features such as the observed surface enrichments at different metallicities as well as the the blue to red supergiant ratio in the SMC. These good agreements give some support to the chosen set of

\footnotetext{
1 The tables can be found at the web address: http://www.unige.ch/sciences/astro/an/; choose on the left "RESEARCH GROUPS", then under the title "EVOLUTION STELLAIRE", choose "ETOILES MASSIVES", then "tables des resultats des recents modeles avec rotation".
} 
physical ingredients; thirdly we think that, in this first extensive study of the effects of rotation on the WR formation at different metallicity, it is necessary to focus on the effects of rotation alone, all other physical ingredients being kept the same. In the future, improvements of the physical ingredients of the models will change some of the results but likely the effects of rotation discussed here will qualitatively remain the same. In that respect it is interesting to mention that in Meynet (2000) we deduced from a previous grid of Wolf-Rayet stellar models at solar metallicity (Paper V) the theoretical value for the number ratio of WR to O-type stars. The results obtained $(\mathrm{WR} / \mathrm{O}=$ 0.026 for $v_{\text {ini }}=0 \mathrm{~km} \mathrm{~s}^{-1}$ and 0.072 for $v_{\text {ini }}=300 \mathrm{~km} \mathrm{~s}^{-1}$ ) are very similar to those obtained from the present grid $(\mathrm{WR} / \mathrm{O}=$ 0.02 for $v_{\text {ini }}=0 \mathrm{~km} \mathrm{~s}^{-1}$ and 0.07 for $v_{\text {ini }}=300 \mathrm{~km} \mathrm{~s}^{-1}$, see Table 3), despite the fact that the two sets of stellar models differ in many respects. Among other things, they differ by the prescription for the mass loss rates, the amount of overshooting and the form of the shear diffusion coefficient. In our view, the nearly identical results obtained in the two cases supports the idea that the results concerning the WR populations are not very sensitive to small changes of these parameters.

\section{Evolution of the rotational velocities at various metallicities}

As discussed in Paper X, the evolution of the rotational velocities at the stellar surface depends mainly on two factors, the internal coupling and the mass loss. The internal coupling is achieved in radiative zones by meridional circulation and by shear diffusion. During the Main Sequence phase, the internal coupling transports angular momentum from the contracting core to the expanding envelope and thus maintains the surface angular velocity above the value it would have in case of nocoupling i.e. of local conservation of the angular momentum. Mass loss on the other hand removes the angular momentum contained in the ejected outer surface layers. Thus, the two effects act in opposite directions for the evolution of the surface velocities. The stronger the coupling mechanisms, the higher the values of the surface velocity at a given stage. The stronger the mass loss rates, the faster the decline of the surface velocities. Let us add that polar winds may decrease the quantity of angular momentum lost by stellar winds; however, as recalled in Sect. 2, we did not take account of this effect due to the moderate initial velocities considered.

How do these two processes, internal coupling and mass loss, depend on the initial metallicity? For what concerns mass loss, it is now well established both theoretically and observationally that the lower the metallicity, the lower the mass loss rates (Kudritzki \& Puls 2000; Vink et al. 2001). This metallicity effect alone would produce a slower decline of the surface velocity at lower metallicity. Now what about the metallicity dependence of the internal coupling mechanisms? At lower metallicities, stars are more compact, and thus the densities in their outer layers at a given stage are higher. Since meridional circulation is more efficient than shear for the transport of angular momentum (Meynet \& Maeder 2000), and since in the outer layers the driving effect for the meridional velocity is the "Gratton-Öpik" term which is proportional to the inverse of the density, the outward transport of angular momentum in these layers will be less efficient at lower than at higher metallicity. This clearly reduces the internal coupling, and weakens the value of the surface velocity obtained at a given stage, all other things being equal. Thus when metallicity decreases, on one side, less angular momentum is removed by stellar winds, and on the other side, less angular momentum is brought up to the surface by the internal transport processes. Taken together these two effects tend to decrease the quantity of angular momentum removed by stellar winds at low metallicity (see also Maeder \& Meynet 2001, Paper VII).

For the evolution of the surface velocities, things are a little more complicated: at lower metallicity, the metallicity dependence of mass loss rates favours higher values for the surface velocities at a given stage. On the other hand, the less efficient internal coupling favours lower values for the surface velocities. The numerical models of the present work show that for the most massive stars $\left(M>30 M_{\odot}\right)$, the effect of the metallicity dependence of the mass loss rates dominates. This can be seen for instance in the case of the $40 M_{\odot}$ tracks plotted in Fig. 1: at high metallicity $(Z=0.040)$ the surface velocity rapidly declines during the core $\mathrm{H}$-burning phase, while at low metallicity $(Z=0.004)$ the star keeps a high surface velocity during most of this phase.

The evolution of the ratio $\Omega / \Omega_{\mathrm{c}}$ of surface angular velocity to the break-up or critical angular velocity is also very different at high and low metallicity. At a low metallicity, the ratio $\Omega / \Omega_{\mathrm{c}}$ at the surface of the $60 M_{\odot}$ model remains near the value of 0.5 during a great part of the Main Sequence phase, while at high metallicity the ratio continuously decreases.

As was already emphasized in Paper X, we again stress that any comparison between observed and predicted rotation for the large masses $\left(M>30 M_{\odot}\right)$ is really much more a test bearing on the mass loss rates than a test of the internal coupling and evolution of rotation. For instance, the increase of $\Omega / \Omega_{c}$ at low metallicity was more pronounced in our previous low metallicity grids (Papers VII and VIII). This is because the mass loss rates adopted in these previous grids were lower by about a factor of two than the new values adopted here. This well illustrates both the uncertainties still pertaining to the mass loss rates and the sensitivity of the evolution of the surface velocity on this still not very well known physical ingredient of the stellar models.

\subsection{Evolution of the rotational velocities during the WR stages}

From Fig. 1 one can see that after the entry into the WR phase, the surface velocity can show rapid changes. These abrupt variations of the surface velocities are linked to rapid changes in the position of the star in the Hertzsprung-Russel diagram caused by surface composition modifications induced by both rotational diffusion and mass loss by stellar winds.

Figure 2 shows specifically the evolution during the WR stages of the rotation period $P(2 \pi / \Omega)$, of the rotation velocities $v$ at the equator and of the fraction $\frac{\Omega}{\Omega_{\mathrm{c}}}$ of the angular velocity to the critical angular velocity at the surface of a star 


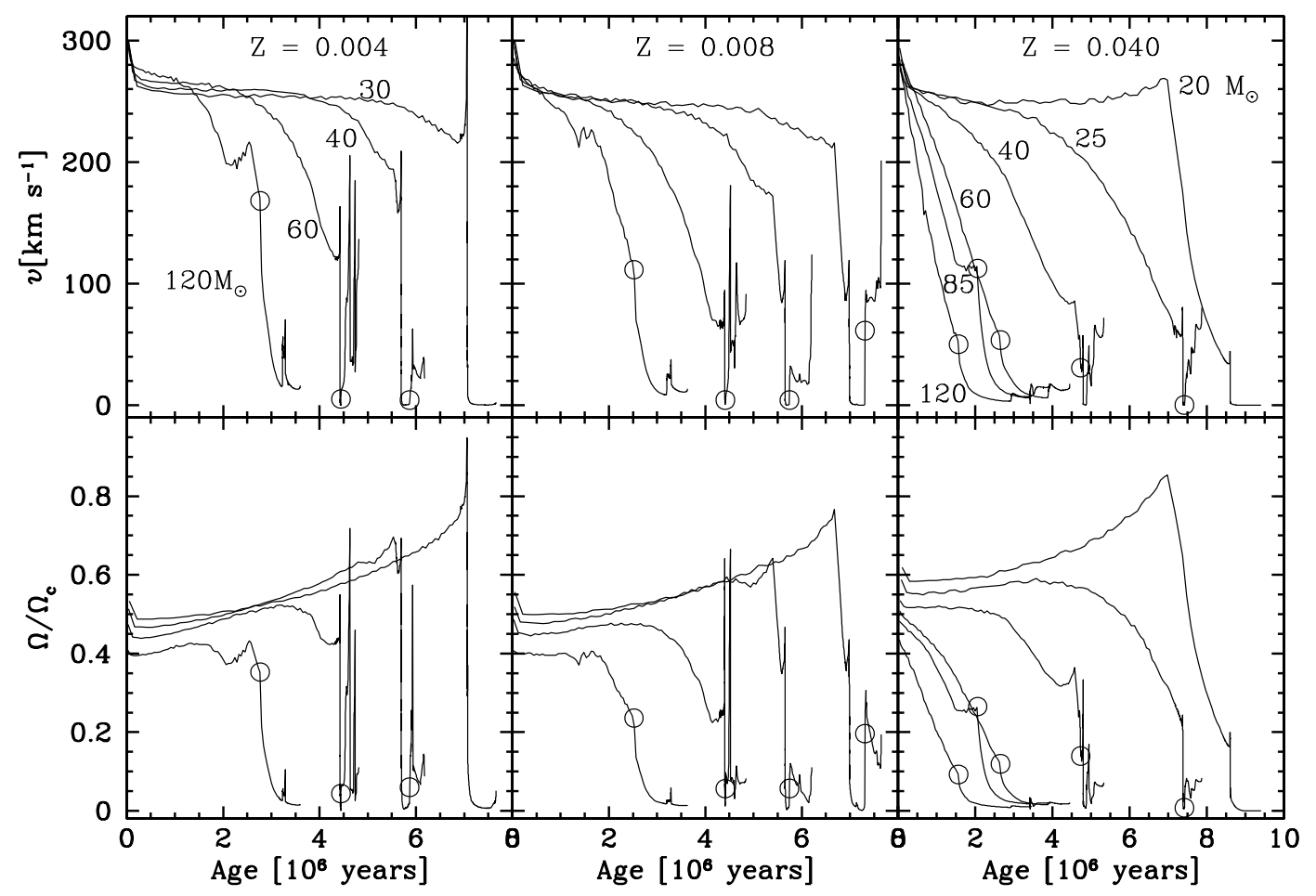

Fig. 1. Evolution of the equatorial velocities (upper panels) and of the ratio $\frac{\Omega}{\Omega_{\mathrm{c}}}$ of the angular velocity to the critical angular velocity (lower panels) at the surface of star models of different initial masses and metallicities. Values of the initial masses are indicated in the upper left and right panels. The initial masses corresponding to the tracks plotted in the middle panels are the same as those shown in the left panels. The initial rotational velocity of all the models is $300 \mathrm{~km} \mathrm{~s}^{-1}$. Empty circles are placed at the stage when the star enters into the Wolf-Rayet phase.

model with an initial mass of $60 M_{\odot}$ and $v_{\text {ini }}=300 \mathrm{~km} \mathrm{~s}^{-1}$ at low and high metallicities (left panels, $Z=0.004$; right panels, $Z=0.040)$. One sees that at low metallicity, much greater and more rapid changes of the surface velocities are expected than at higher metallicity. Thus the encounter of the break-up limit during the WR phase may appear more often at lower metallicity (see the lower panels in Fig. 2). This comes from the fact that at low metallicity the whole WR phase occurs while the star is in the core He-burning phase, a phase during which our most massive star models experience strong changes in radius. In contrast, at $Z=0.040$, nearly the whole eWNL phase occurs during the core H-burning phase (for a definition of the eWNL phase see Sect. 5.1). One sees that the high mass loss rates undergone by the star makes the surface velocity to decrease regularly. Only when the star has lost its complete H-rich envelope (i.e. when the star enters the eWNE stage in terms of evolutionary models), does strong contraction of the surface layers, caused by the decrease of the opacity in the outer regions, produces a small increase of the surface velocity. We see that despite the strong increase of $\Omega$ the velocity $v$ and $\frac{\Omega}{\Omega_{c}}$ do not change very much, since the radius is much smaller in the eWNE stage. All the transitions in the WR stage are fast, thus the transfer of angular momentum by meridional circulation is limited and the evolution of the rotation at the surface is dominated by the local conservation of angular momentum, which explains the fast and large decreases of $P$ or $\Omega$.

One notes that in general the final surface velocity at low metallicity is also higher than at high metallicity, a fact which may be easily explained as a consequence of the lower mass loss rates at lower metallicity. From Table 1, one sees also that at a given metallicity the velocities obtained at the end of the He-burning phase for the WR stars are in general smaller for the higher initial mass stars. This results from the fact that the higher the initial mass, the larger the amount of mass (and thus of angular momentum) removed by the stellar winds.

The implications of the present models for the rotation rates of pulsars at birth and the possible progenitors of the collapsar models will be considered in a forthcoming paper. Let us just mention here that most of the WR stellar models have enough specific angular momentum in their core to be good candidates for collapsar models. On the other hand, if no angular momentum is lost during the core-collapse supernova explosion, these models would predict very fast rotational velocities for the neutron stars at birth. These results are in qualitative agreement with those obtained by Heger \& Woosley (2004), and Woosley $\&$ Heger (2003) in the case of no magnetic field.

\section{Evolutionary tracks, lifetimes and final masses}

In Fig. 3 some evolutionary tracks of rotating models are shown. Note that during the WR phase the values of the effective temperature used to draw the tracks take account of the non-negligible optical thickness of the winds (see Sect. 1). At high metallicity and for the most massive stars, the entry into the WR phase occurs during the MS phase (see Sect. 5 below). Therefore the non-WR part of the track is quite short. For lower initial mass stars, rotation makes the tracks overluminous and more extended towards lower effective temperatures during the 


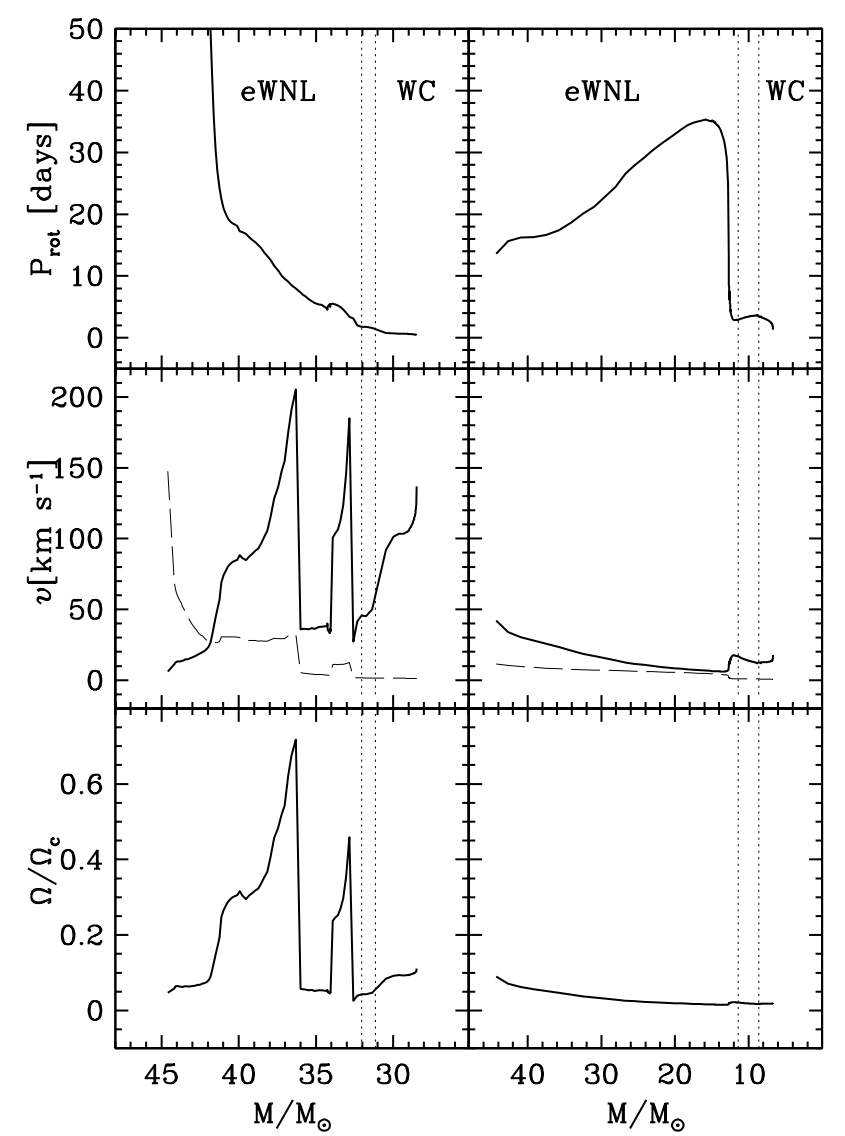

Fig. 2. Evolution as a function of the actual mass of the rotation period, of the surface equatorial velocity and of the ratio of the angular velocity to the critical value during the WR stage of rotating stars. The long-dashed lines in the panels for the velocities show the evolution of the radius in solar units. Left: the WR phase of a star with an initial mass of $60 M_{\odot}$ with $v_{\text {ini }}=300 \mathrm{~km} \mathrm{~s}^{-1}$ and $Z=0.004$. Right: for an initial mass of $60 M_{\odot}$ with $v_{\text {ini }}=300 \mathrm{~km} \mathrm{~s}^{-1}$ and $Z=0.040$.

MS phase. This was also the case in previous works (see Heger \& Langer 2000; Meynet \& Maeder 2000, 2003). Note however that for very fast rotation the star may be so efficiently mixed that it has a nearly homogeneous evolution and would present a very blue track during the MS phase (Maeder 1987). This is the case for the $500 \mathrm{~km} \mathrm{~s}^{-1} 60 M_{\odot}$ stellar model at $Z=0.004$ in the present grid (see Table 1). Let us recall also that at low metallicity, for those stars which do not already enter the WR phase during the MS phase, the evolution towards the red supergiant stage is favoured when rotation is included in the stellar model (Maeder \& Meynet 2001).

Table 1 presents some properties of the models. Columns 1 and 2 give the initial mass and the initial velocity $v_{\text {ini }}$ respectively. The mean equatorial rotational velocity $\bar{v}$ during the MS phase is indicated in Col. 3. This quantity is defined as in Paper V. The H-burning lifetimes $t_{\mathrm{H}}$, the lifetimes as an O-type star on the MS $t_{\mathrm{O}}$ (we assumed that O-type stars have an effective temperature higher than about $33000 \mathrm{~K}$ ), the masses $M$, the equatorial velocities $v$, the helium surface abundance $Y_{\mathrm{s}}$ and the surface ratios (in mass fraction) N/C and N/O at the end of the H-burning phase are given in Cols. 4 to 10. Columns 11 to 17 present some characteristics of the stellar models at the end of the He-burning phase, $t_{\mathrm{He}}$ is the He-burning lifetime. More details on the models are given in the electronic tables (see Sect. 2).

From Table 1 one sees that for $Z=0.040$ the MS lifetimes are increased by about $16-27 \%$ when the initial rotational velocity increases from 0 to $300 \mathrm{~km} \mathrm{~s}^{-1}$. Similar increases were found at solar metallicity (see Papers V and X). The He-burning lifetimes are increased by rotation for the 60 and $120 M_{\odot}$ models by respectively 12 and $34 \%$. This comes from the fact that the most massive stars already enter the WR phase during the core H-burning phase. The strong mass losses that they experienced at the end of the H-burning phase produce small $\mathrm{He}$-cores at the beginning of the core He-burning phase. This tends to reduce the central temperatures and to increase the He-burning lifetimes.

Rotation decreases the He-burning lifetime for the $25 M_{\odot}$ by $8 \%$ at solar metallicity and by $17 \%$ at $Z=0.040$. Contrary to what happens in the high mass star range, in the rotating $25 M_{\odot}$ stellar model the He-core at the end of the MS phase is significantly more massive than in the non-rotating model (by $42 \%$ at $Z=0.040$ ) and thus the rotating track is much more luminous during most of the He-burning phase than the non-rotating one. This of course tends to reduce the duration of the core He-burning phase.

As indicated in Sect. 2, the present stellar models were computed with the mass loss rates of Vink et al. (2000). This prescription shows a dramatic increase of the mass loss rates for OB Main-Sequence stars when they cross, from blue to red, the bistability limit which occurs at an effective temperature equal to $\sim 25000 \mathrm{~K}$. The increase is due to a drastic change in the ionization of the wind. To illustrate this effect, the evolution, during the Main-Sequence phase, of the mass of our stellar models is represented in Fig. 4 as a function of $T_{\text {eff. }}$. When the star models cross the bistability limit, the tracks turn down very abruptly in the cases of the most massive stars, more smoothly in the cases of smaller initial mass stars. This results from the important increase of the mass loss rates, by about 1 dex, when the star crosses the bistability limit. As an example, the $25 M_{\odot}$ stellar model, which spends only $11 \%$ of its MS lifetime on the red side of the bistability limit, loses during this short phase more than $60 \%$ of the mass lost during the whole MS phase. For stars more massive than about $60 M_{\odot}$ and less massive than about $10 M_{\odot}$, there is no jump in the mass loss rates, since their tracks remain on one side of the bistability limit during the whole Main-Sequence phase.

One can wonder to what extent the bistability effect represents an important feature favouring the entry of the stars in the WR regime. Let us just remark here that this effect alone is of little help in that respect. Indeed models without rotation but taking account of this bistability effect give a poor fit of the observed variation of the number ratio of WR to O-type stars with the metallicity (see Fig. 10), while rotating models much better reproduce the observed data (see Sect. 6).

From Table 1, comparing the final masses obtained at $Z=$ 0.040 for the rotating and the non-rotating model, one can see that rotation produces in general smaller final masses. Figure 5 shows the final masses obtained for different initial masses and metallicities. At $Z=0.040$, most of the stars in the mass range 


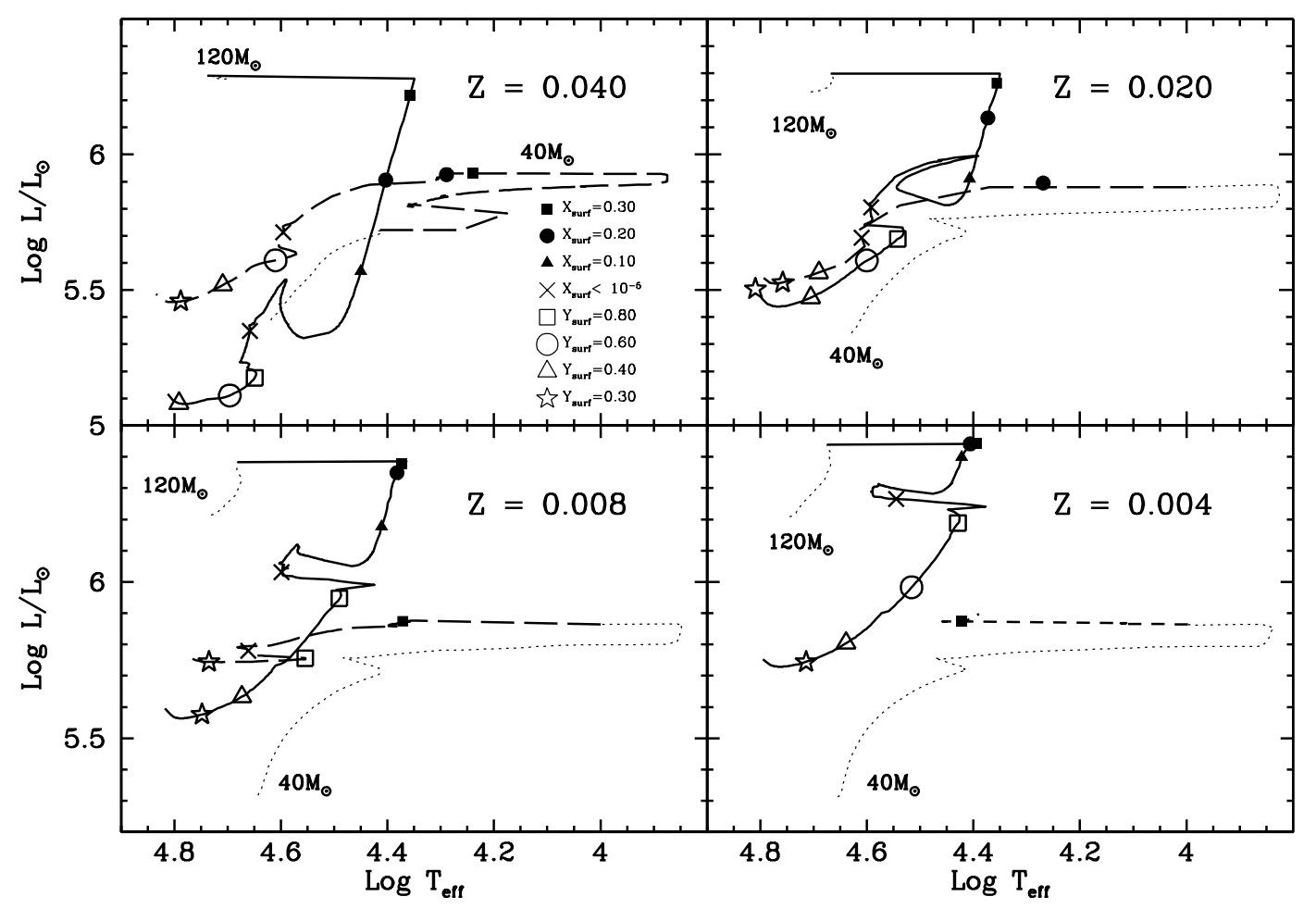

Fig. 3. Evolutionary tracks for 40 and $120 M_{\odot}$ rotating models at different metallicities. The initial velocity is $300 \mathrm{~km} \mathrm{~s}^{-1}$. The light dotted lines correspond to the non-WR part of the tracks. The tracks during the WR phase are shown by heavy lines (continuous for the $120 M_{\odot}$ and dashed for the $40 M_{\odot}$ model). Symbols along the tracks are placed where the indicated surface hydrogen $\left(X_{\text {surf }}\right)$ and helium $\left(Y_{\text {surf }}\right)$ abundances are reached.

considered here end their life with masses below $10 M_{\odot}$. All stars with masses above about $50 M_{\odot}$ reach a final mass between 5 and $7.5 M_{\odot}$. In contrast, at a metallicity one order of magnitude smaller, the final masses of stars are significantly higher, being in the range between 17 and $29 M_{\odot}$ for stars with initial masses above $60 M_{\odot}$. This well illustrates the effect of the metallicity dependence of the mass loss rates, an effect which is qualitatively similar to that found in non-rotating stellar models (Maeder 1991).

\section{Rotating models of Wolf-Rayet stars}

\subsection{Effects of rotation and metallicity on the evolutionary scenarios leading to the formation of WR stars}

The effects of rotation on the evolution of massive single stars into the Wolf-Rayet phase have been discussed by Maeder (1987), Fliegner and Langer (1995), Maeder \& Meynet (2000b) and Meynet (2000). These studies were mainly based on solar metallicity models. Here we extend these discussions to lower and higher than solar metallicities.

We had to choose a set of criteria to decide when a stellar model enters the WR phase. Ideally, of course, the physics of the models should determine when the star is a WR star. However our poor knowledge of the physics involved, as well as the complexity of models coupling the stellar interiors to the winds, are such that this approach is not yet possible. Instead, it is necessary to adopt some empirical criteria for deciding when a star becomes a WR star. In this work the star enters the WR phase when two conditions are fullfilled: 1) $\log T_{\text {eff }}>4.0$ (note that after the entry into the WR phase the star may exceptionally spend very short periods in cooler regions of the HR diagram. In general however, the whole WR phase is spent at effective temperatures well above $\log T_{\text {eff }}=4.0$ ); 2) the mass fraction of hydrogen at the surface $X_{\mathrm{s}}$ is inferior to 0.4 . Reasonable changes to these values (for instance adopting $X_{\mathrm{s}}<$ 0.3 instead of 0.4 ) do not affect the results significantly. Let us recall that the effective temperature considered here is an effective temperature appropriately averaged over the surface (see Meynet \& Maeder 1997). We consider the WR star to be of the eWNL type when the mass fraction of hydrogen at the surface is superior to $10^{-5}$, (we adopt here the denomination proposed by Foellmi et al. 2003b for the WNL and WNE phase, based on evolutionary criteria instead of spectroscopic ones, hence the small "e" before the name). The eWNE phase begins at the end of the eWNL phase, while the transition WN/WC phase is considered to begin when the mass fraction of carbon at the surface becomes superior to $10 \%$ of the mass fraction of nitrogen. Both the eWNE and the transition WN/WC phases end when the WC/WO phase begins, which we assume to begin when the mass fraction of nitrogen at the surface becomes less than $10 \%$ of the mass fraction of carbon. We shall not distinguish here between WC and WO stars. Also, changes of the numerical values of the limits have very little consequence since the transitions are fast.

Internal mixing favours the entry into the WR phase in two ways, firstly by allowing chemical species produced in the core 
Table 1. Properties of the stellar models at the end of the H-burning phase and at the end of the He-burning phase. The masses are in solar mass, the velocities in $\mathrm{km} \mathrm{s}^{-1}$, the lifetimes in million years and the abundances in mass fraction.

\begin{tabular}{|c|c|c|c|c|c|c|c|c|c|c|c|c|c|c|c|c|}
\hline \multirow[t]{2}{*}{$M$} & \multirow[t]{2}{*}{$v_{\text {ini }}$} & \multirow[t]{2}{*}{$\bar{v}$} & \multicolumn{7}{|c|}{ End of H-burning } & \multicolumn{7}{|c|}{ End of He-burning } \\
\hline & & & $t_{\mathrm{H}}$ & $t_{\mathrm{O}}$ & $M$ & $v$ & $Y_{\mathrm{s}}$ & $\mathrm{N} / \mathrm{C}$ & $\mathrm{N} / \mathrm{O}$ & $t_{\mathrm{He}}$ & $M$ & $v$ & $Y_{\mathrm{s}}$ & $\mathrm{C}$ & $\mathrm{N}$ & $\mathrm{O}$ \\
\hline & & & \multicolumn{14}{|c|}{$Z=0.004$} \\
\hline 120 & 300 & 244 & 3.232 & 2.770 & 52.930 & 23.9 & 0.98 & 42.3 & 60.0 & 0.362 & 17.178 & 16.5 & 0.24 & 0.46 & 0 & 0.29 \\
\hline 60 & 300 & 236 & 4.422 & 4.206 & 51.824 & 157.0 & 0.41 & 5.33 & 1.82 & 0.383 & 28.465 & 136.8 & 0.14 & 0.34 & 0 & 0.51 \\
\hline $60^{1}$ & 500 & 392 & 5.103 & 4.657 & 36.640 & 65.7 & 0.93 & 45.1 & 43.6 & 0.400 & 12.359 & 48.1 & 0.29 & 0.49 & 0 & 0.22 \\
\hline 40 & 300 & 249 & 5.690 & 5.114 & 36.755 & 209.3 & 0.29 & 1.64 & 0.53 & 0.476 & 22.333 & 22.0 & 0.74 & $4 e-5$ & $252 \mathrm{e}-5$ & $14 \mathrm{e}-5$ \\
\hline \multirow[t]{2}{*}{30} & 300 & 250 & 7.059 & 6.196 & 28.654 & 336.4 & 0.25 & 0.99 & 0.31 & 0.590 & 18.866 & 2.59 & 0.63 & $9 e-5$ & $218 \mathrm{e}-5$ & $47 e-5$ \\
\hline & & & \multicolumn{14}{|c|}{$Z=0.008$} \\
\hline 120 & 300 & 222 & 3.199 & 2.522 & 36.612 & 12.4 & 0.96 & 46.5 & 52.2 & 0.427 & 13.393 & 13.8 & 0.25 & 0.47 & 0 & 0.27 \\
\hline 60 & 300 & 209 & 4.401 & 4.255 & 48.447 & 88.6 & 0.51 & 9.85 & 3.3 & 0.435 & 16.446 & 91.7 & 0.19 & 0.41 & 0 & 0.39 \\
\hline 40 & 300 & 233 & 5.651 & 4.928 & 35.294 & 11.6 & 0.34 & 2.59 & 0.8 & 0.550 & 17.342 & 123.9 & 0.26 & 0.30 & $6.9 \mathrm{e}-4$ & 0.43 \\
\hline \multirow[t]{2}{*}{30} & 300 & 240 & 6.982 & 5.817 & 27.457 & 119.2 & 0.28 & 1.39 & 0.4 & 0.658 & 12.106 & 201.2 & 0.59 & 0.21 & $2.9 \mathrm{e}-3$ & 0.19 \\
\hline & & & \multicolumn{14}{|c|}{$Z=0.040$} \\
\hline 120 & 0 & 0 & 2.533 & 1.823 & 18.569 & 0 & 0.94 & 52.5 & 25.8 & 0.467 & 8.572 & 0 & 0.37 & 0.46 & 0 & 0.12 \\
\hline 120 & 300 & 157 & 2.929 & 1.565 & 13.954 & 4.9 & 0.93 & 56.1 & 23.5 & 0.525 & 7.112 & 9.0 & 0.39 & 0.46 & 0 & 0.10 \\
\hline $120^{2}$ & 300 & 157 & 3.182 & 1.565 & 9.595 & 4.0 & 0.92 & 60.1 & 22.4 & 0.673 & 4.846 & 7.9 & 0.62 & 0.30 & 0 & 0.03 \\
\hline 85 & 300 & 180 & 3.413 & 2.061 & 15.592 & 8.7 & 0.89 & 58.8 & 19.4 & 0.525 & 7.295 & 18.1 & 0.38 & 0.46 & 0 & 0.11 \\
\hline $85^{2}$ & 300 & 180 & 3.560 & 2.061 & 11.633 & 7.2 & 0.88 & 61.8 & 18.6 & 0.746 & 4.7087 & 16.6 & 0.42 & 0.44 & 0 & 0.09 \\
\hline 60 & 0 & 0 & 3.122 & 2.213 & 29.272 & 0 & 0.73 & 73.4 & 10.8 & 0.410 & 11.329 & 0 & 0.30 & 0.47 & 0 & 0.18 \\
\hline 60 & 300 & 176 & 3.894 & 2.652 & 12.684 & 8.9 & 0.93 & 57.3 & 20.1 & 0.549 & 6.686 & 17.8 & 0.43 & 0.44 & 0 & 0.08 \\
\hline $60^{2}$ & 300 & 176 & 4.080 & 2.652 & 9.292 & 7.1 & 0.92 & 59.9 & 19.5 & 0.682 & 4.772 & 14.6 & 0.65 & 0.28 & 0 & 0.02 \\
\hline 40 & 300 & 187 & 4.793 & 4.073 & 29.948 & 52.7 & 0.57 & 10.7 & 2.3 & 0.526 & 11.418 & 71.9 & 0.27 & 0.46 & 0 & 0.22 \\
\hline $40^{2}$ & 300 & 187 & 4.792 & 4.073 & 29.501 & 40.0 & 0.57 & 10.9 & 2.3 & 0.503 & 9.036 & 62.3 & 0.27 & 0.47 & 0 & 0.20 \\
\hline 25 & 0 & 0 & 5.815 & 4.527 & 23.503 & 0 & 0.32 & 0.31 & 0.11 & 0.585 & 13.871 & 0 & 0.52 & $3.2 \mathrm{e}-4$ & $1.8 \mathrm{e}-2$ & $9.9 \mathrm{e}-3$ \\
\hline 25 & 300 & 205 & 7.376 & 5.807 & 21.318 & 74.8 & 0.46 & 3.2 & 0.9 & 0.484 & 9.588 & 80.2 & 0.33 & 0.47 & 0 & 0.15 \\
\hline 20 & 300 & 227 & 8.602 & 3.105 & 16.927 & 43.7 & 0.38 & 1.6 & 0.5 & 0.792 & 9.245 & 0.04 & 0.49 & $1.3 \mathrm{e}-3$ & $1.4 \mathrm{e}-2$ & $1.3 \mathrm{e}-2$ \\
\hline
\end{tabular}

${ }^{1}$ Model computed with anisotropic stellar winds and with an $\alpha$-enhanced mixture of the heavy elements.

${ }^{2}$ Model computed with mass loss rates during the WR phase dependent on the metallicity (Crowther et al. 2002).

to diffuse in the radiative envelope and, secondly, by making the mass of the convective core larger (see also Meynet \& Maeder 2000). In the non-rotating model, mass loss by stellar winds is the key physical ingredient which allows internal chemical products to appear at the surface and thus form a WR star. The star becomes a WR star only when sufficiently deep layers are uncovered.

In Fig. 6 the evolution of the structures for $60 M_{\odot}$ models with and without rotation at twice the solar metallicity are shown. In the case of the $60 M_{\odot}$ model, the most striking differences between the non-rotating and the rotating model are the following:

- Inclusion of rotation in the models allows an earlier entry into the WR phase. Typically here in the non-rotating model the star becomes a WR star when the actual mass is $34.3 M_{\odot}$ and the mass fraction of $\mathrm{H}$ at the centre $X_{\mathrm{c}}$ is 0.04 , while in the rotating model it enters the WR phase when the mass is equal to $44.9 M_{\odot}$ and $X_{\mathrm{c}}$ is equal to 0.24 .

- As a consequence the WR lifetime as well as the duration of the eWNL phase will be increased by rotation. Also the actual luminosity at the entry of the WR phase will be higher for the rotating models.

- The duration of the WC phase is reduced.

Qualitatively similar effects of rotation were obtained at solar metallicity (cf. Paper X) except for the effect of rotation on the WC phase: at solar metallicity and for the $60 M_{\odot}$ stellar model the WC phase is slightly enhanced by rotation (see Table 2 in Paper $X$ ). At $Z=0.004$, the WC phase becomes longer when the initial rotation increases (see Table 3 ). In addition to the above effects, rotation decreases the minimum initial mass of single stars going through a WR phase (see Fig. 8) and the duration of the transition WN/WC phase is much longer in the rotating models than in the non-rotating ones (see Table 3 ).

As said in Sect. 2, the importance of these effects depends on the values adopted for the initial velocity and on various physical ingredients of the stellar models as for instance the mass loss rate and/or the overshooting. Some illustration of the effects of different values of rotation for $60 M_{\odot}$ models at solar metallicity can be found in Meynet (1999) and in Paper X. Results for a fast rotating $60 M_{\odot}$ stellar model at $Z=0.004$ $\left(v_{\text {ini }}=500 \mathrm{~km} \mathrm{~s}^{-1}\right)$ are presented in Tables 1 and 3 . In that last 


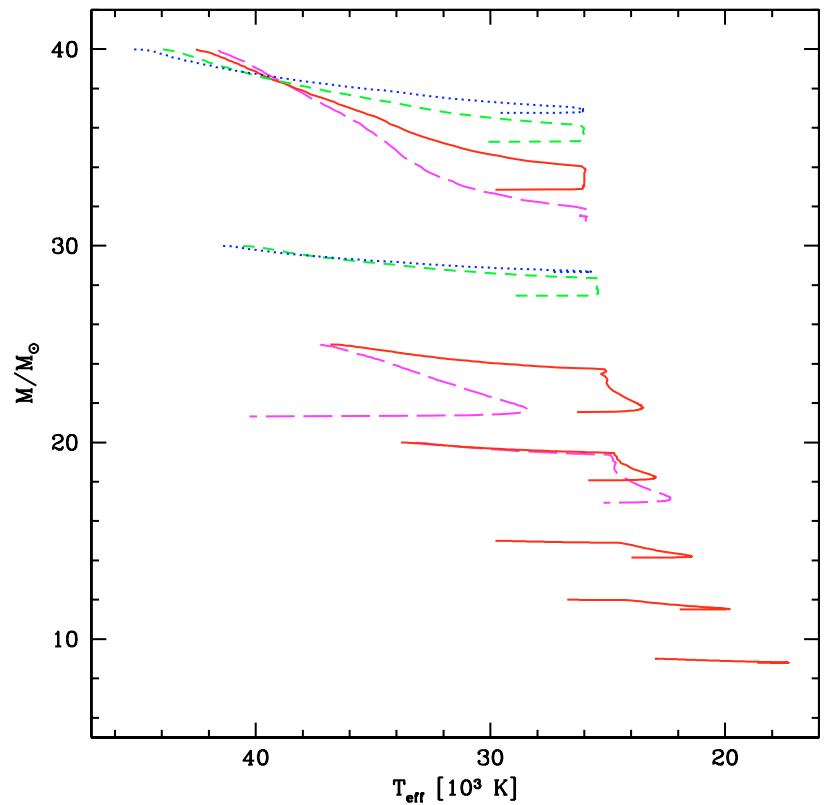

Fig. 4. Evolution of the mass of the stars as a function of the effective temperature for different initial mass models (from 9 to $40 M_{\odot}$ ) at various metallicities during the Main-Sequence phase $\left(v_{\text {ini }}=300 \mathrm{~km} \mathrm{~s}^{-1}\right)$. The initial mass of the stars is the ordinate of the hottest point of each track (left point). Models at $Z=0.004,0.008,0.020$ and 0.040 are shown with dotted, short-dashed, continuous and long-dashed lines respectively. One can note the effect of the crossing of the bistability limit around $25000 \mathrm{~K}$ (see text).

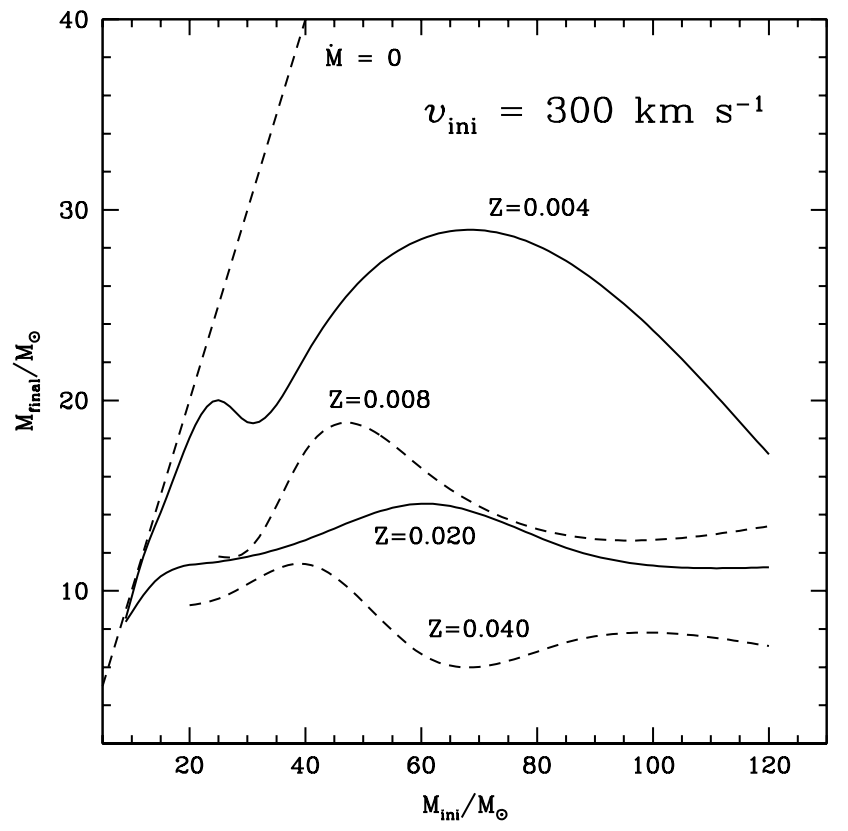

Fig. 5. Relations between the final and the initial mass for rotating stellar models at various metallicities. The line with slope one, labeled $\dot{M}=0$, corresponds to the case without mass loss.

example, we see that increasing the initial velocity from 300 to $500 \mathrm{~km} \mathrm{~s}^{-1}$ more than doubles the WR lifetime. This enhancement results from the more efficient internal mixing at high rotation and not to the increase of the mass loss rate due to rotation.

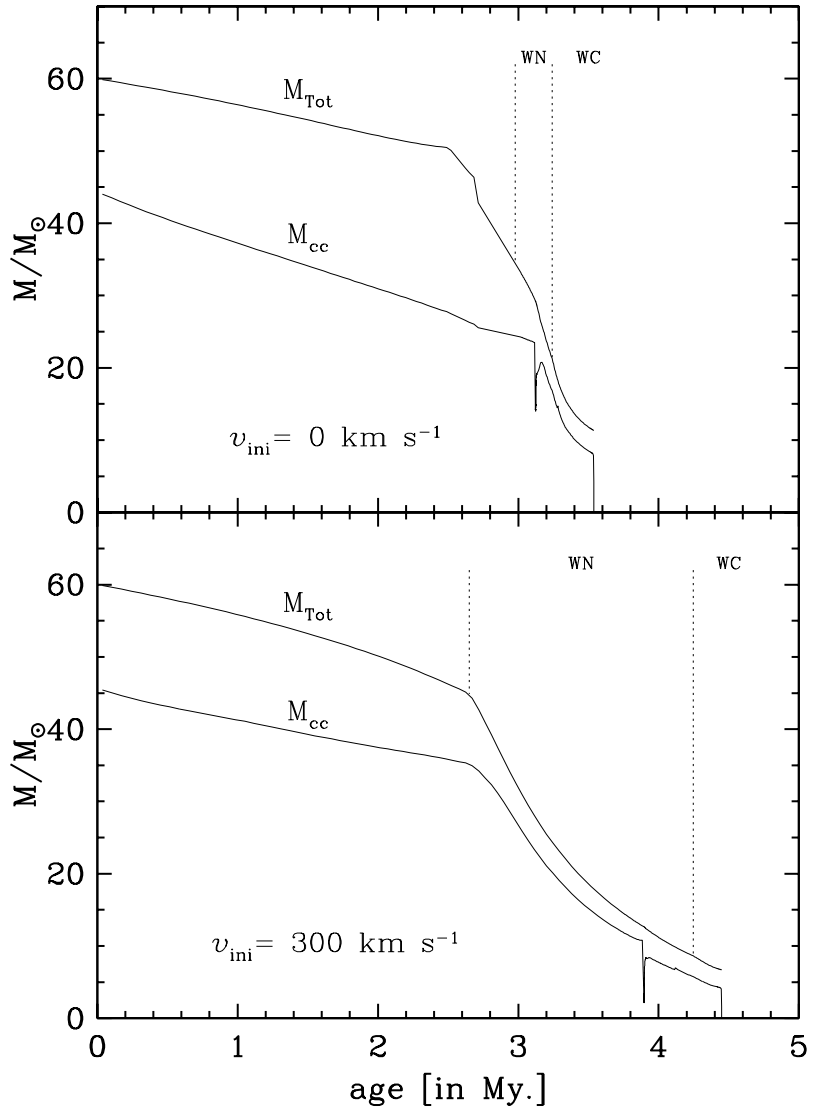

Fig. 6. Evolution as a function of time of the total mass $M_{\text {Tot }}$ and of the mass of the convective cores $M_{\mathrm{cc}}$ during the $\mathrm{H}$ - and He-burning phases, for a $60 M_{\odot}$ stellar model with and without rotation at $Z=$ 0.040. The type of the WR star at a given age is given in the upper part of the figure.

From the present rotating models one can derive two interesting limiting masses. The first, $M_{\mathrm{OWR}}$, is the minimum initial mass of a single star entering the WR phase during the MS phase. The second, $M_{\mathrm{WR}}$, is the minimum initial mass of a single star entering the WR phase at any point in the course of its lifetime. These two limiting masses define the mass ranges of three evolutionary scenarios for the massive stars:

- For $M>M_{\mathrm{OWR}}$, the stars will avoid the Luminous Blue Variable stage after the MS phase. In this case, they will go through the following phases: O-eWNL-eWNE-WC/WO. Note that here we assume that once the star has entered the WR regime, it remains a WR star for the rest of its lifetime. Some stars however may evolve in cooler regions of the HR diagram after they have entered the WR phase and might thus encounter the Humphreys-Davidson limit. These star models would present characteristics similar to LBV stars and could thus belong to this category.

- For $M_{\mathrm{WR}}<M<M_{\mathrm{OWR}}$, after the MS phase, the star will evolve into the cooler part of the HR diagram, where it may encounter the $\Omega \Gamma$-limit (Maeder \& Meynet 2000a) or become a Red Supergiant. In that case, one would have O-LBV or RSG-eWNL-eWNE-WC/WO. Evolution may not necessarily proceed up to the WC/WO stage, it may stop at the eWNE or eWNL stage. 
Table 2. Mass limits for the different evolutionary scenarios (see text).

\begin{tabular}{cccc}
\hline \hline$Z$ & $\begin{array}{c}v_{\text {ini }} \\
{\left[\mathrm{km} \mathrm{s}^{-1}\right]}\end{array}$ & $\begin{array}{c}\mathrm{M}_{\mathrm{WR}} \\
{\left[M_{\odot}\right]}\end{array}$ & $\begin{array}{c}\mathrm{M}_{\text {OWR }} \\
{\left[M_{\odot}\right]}\end{array}$ \\
\hline 0.040 & 0 & 29 & 42 \\
0.040 & 300 & 21 & 39 \\
0.020 & 0 & 37 & 62 \\
0.020 & 300 & 22 & 45 \\
0.008 & 300 & 25 & 69 \\
0.004 & 300 & 32 & 75 \\
\hline
\end{tabular}

- For $M<M_{\mathrm{WR}}$, after the core H-burning phase the O-type star will become a supergiant, but it will never enter the WR phase. The blue or red nature of the supergiant depends among other parameters on rotation (Maeder \& Meynet 2001). The evolutionary sequence in that case will be O-RSG/BSG. Blue loops may be present especially in the lower mass star range.

In order to estimate $M_{\mathrm{OWR}}$ and $M_{\mathrm{WR}}$ we interpolated between corresponding evolutionary stages of the tracks using the logarithm of the initial mass as the interpolating factor. We obtain the mass limits indicated in Table 2 . We can note that, at a given metallicity, the mass limits are lower when the rotation rate is higher, as expected from the considerations mentioned above. As we shall see in Sect. 6, these results on the mass limits have interesting consequences for the expected number of WR stars at different metallicity, as well as for the predicted number of type $\mathrm{Ib} / \mathrm{Ic}$ supernovae. One notes also from Table 2 that the mass limits are lower at higher metallicities, as would be the case for models without rotation. This is due to the greater mass loss rates experienced by stars at higher metallicity.

The mass range for which the WR phase is preceded by a LBV and/or a RSG phase shifts to higher values at lower metallicity, it also extends over a larger mass interval. Typically, at $Z=0.040$ rotating models predict that such a scenario for single massive stars occurs in the mass range between 21 and $39 M_{\odot}$, while it occurs in the mass range between 32 and $75 M_{\odot}$ at $Z=0.004$. Since qualitatively a same trend is predicted by the non-rotating models, this result would imply that the upper luminosity of LBV stars should be lower at higher metallicity. However one should be cautious here. First, one cannot discard the possibility that a star enters the WR phase and later undergoes a shell ejection similar to a LBV outburst due for instance to reaching the $\Omega \Gamma$-limit (see Maeder $\&$ Meynet 2000a). Moreover the observed stellar populations are a mixture of stars of different initial velocities, whose initial distributions may depend on the metallicity. This would contribute to the blurring of the schematic picture just described above.

As already stated in Sect. 2, the results shown is Table 2 are sensitive to various physical ingredients. For instance, an enhancement of the core sizes and/or of the initial velocity and/or of the mass loss rates would favour the WR formation and thus the lowering of these mass limits. Thus the numbers shown in Table 2 may undergo some changes in the future when improvements in these physical ingredients will be reached.
However, at least qualitatively, the trend they show, namely the fact that rotation lowers the mass limits, will likely persist. This is well illustrated by the fact that a similar trend was already present in our Paper V models which were computed with different prescriptions for the mass loss rates, the overshooting and the rotational mixing (see Sect. 2).

For a given initial mass and an initial rotational velocity, the greater the metallicity the larger the luminosity range spanned during the WR phase, and a lower minimum luminosity is reached. This can be seen from Fig. 3. Interestingly we can see that at a given metallicity, the higher initial mass stars are in general the progenitors of the less luminous WC stars. This is mainly due to the fact that the mass loss rates increase with the luminosity, enabling the most massive stars to enter the WR phase at an earlier stage and thus to lose mass at high rates during a larger portion of their lifetime. Thus these stars end with small final masses and hence with small luminosities since at this stage they are following a well known mass luminosity relation (Schaerer \& Maeder 1992).

\subsection{The Wolf-Rayet lifetimes}

The WR lifetimes of the present rotating models for the four metallicities are plotted as a function of the initial mass in Fig. 7. As was the case with the non-rotating models (Maeder 1991; Maeder \& Meynet 1994), the metallicity dependence of the mass loss rates is responsible for two features: 1) for a given initial mass and velocity the WR lifetimes are greater at higher metallicities. Typically at $Z=0.040$ and for $M>60 M_{\odot}$ the WR lifetime is of the order of $2 \mathrm{Myr}$, while at the metallicity of the SMC the WR lifetimes in this mass range are between $0.4-0.8 \mathrm{Myr}$; 2) the minimum mass for a single star to evolve into the WR phase is lower at higher metallicity.

It is interesting to compare the WR lifetimes obtained at $Z=0.040$ and 0.004 from different sets of models (see Fig. 8). First, one can notice that the present non-rotating models with the updated mass loss rates give very similar results to those obtained with the "normal" mass loss rates by Meynet et al. (1994; see how the black squares give a good fit to the $Z=$ 0.040 dotted curve). As was discussed in Paper X, rotation has a similar effect as an enhancement of the mass loss rates on the WR lifetimes. Namely, for a given initial mass it increases the WR lifetime and also lowers the minimum initial mass of single stars going through a WR phase. Note however that quantitatively the results obtained from rotating models may be different from the one obtained from the enhanced mass loss rate models. At low metallicity, the curve obtained from the rotating models is always above the one obtained from the enhanced mass loss rate models. More importantly, rotation seems to be much more efficient in lowering the value of $M_{\mathrm{WR}}$. This is due to the fact that rotation helps in forming WR stars through internal mixing, a process which, in contrast to mass loss rates, is important for all massive stars, not only the most massive ones. At high metallicity, the situation is somewhat different, because when metallicity increases the mass loss rate becomes the dominant parameter. 


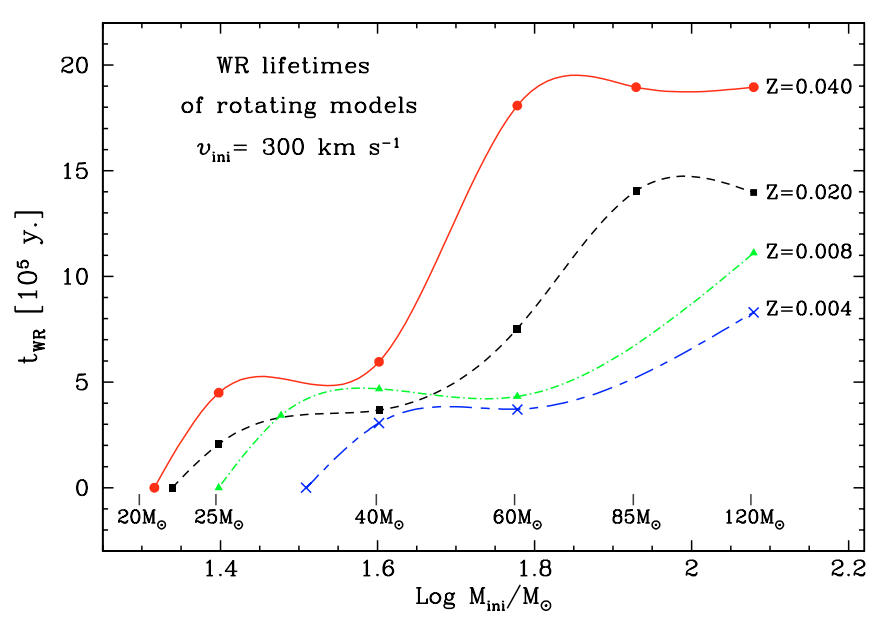

Fig. 7. Lifetimes of Wolf-Rayet stars from various initial masses at four different metallicities. All the models begin their evolution with $v_{\text {ini }}=300 \mathrm{~km} \mathrm{~s}^{-1}$ on the ZAMS, the corresponding time-averaged velocities during the MS O-type star phase are given in Table 1.

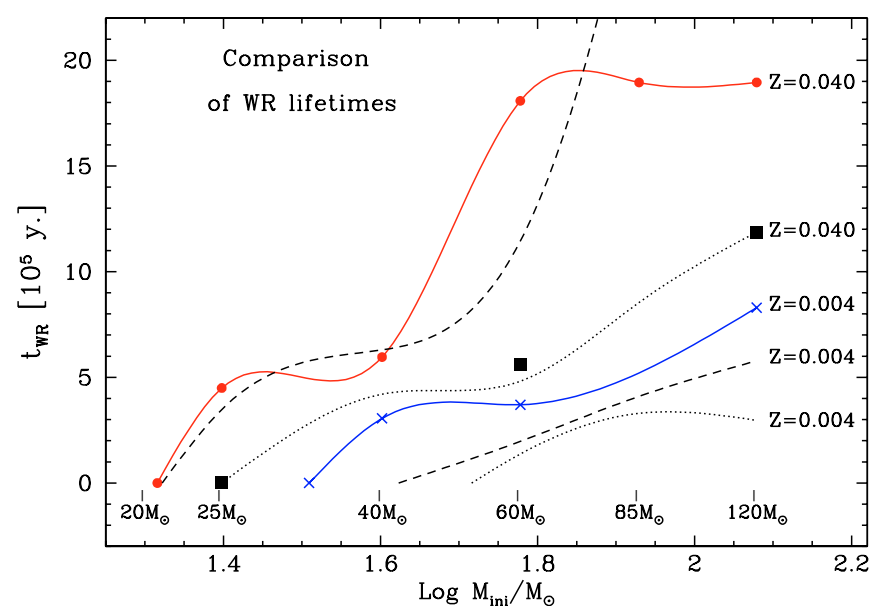

Fig. 8. Lifetimes of Wolf-Rayet stars from various initial masses for $Z=0.040$ and 0.004 from different sets of models: continuous lines are for the present rotating models with $v_{\text {ini }}=300 \mathrm{~km} \mathrm{~s}^{-1}$, the dotted and dashed lines are for the non-rotating stellar models with "normal" and "enhanced" mass loss rates computed by Meynet et al. (1994). The black squares indicate the lifetimes obtained for the non-rotating 25 , 60 and $120 M_{\odot}$ models obtained in the present work at $Z=0.040$.

In Fig. 9, the duration of the different WR subphases is plotted as a function of the initial mass for the various metallicities considered in this work. Only the results with rotation are plotted. The durations of these different subphases are given in Table 3. The greatest part of the WR lifetime is spent in the eWNL phase. Note that, as explained above, rotation increases the duration of this phase. For identical initial rotational velocities, the duration of the eWNL phase is greater at higher metallicity. This results from the metallicity dependence of the mass loss rates. At higher metallicity, the higher mass loss rates by stellar winds enable the star to enter the WR phase at an earlier stage. The eWNE phase is also longer at higher metallicity (as is also the case for non-rotating models). The WC phase keeps more or less the same duration for all the metallicities in the higher mass star range. In the lower mass star range the
Table 3. Lifetimes in Myr of WR stars of different initial masses and metallicities. The durations of various WR subphases are also indicated as well as theoretical predictions for various number ratios.

\begin{tabular}{|c|c|c|c|c|c|c|}
\hline $\bar{M}$ & 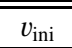 & 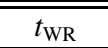 & $\bar{~} t_{\mathrm{eWNL}}$ & $\overline{t_{\text {eWNE }}}$ & $\bar{t}_{\mathrm{WN} / \mathrm{WC}}$ & $\overline{t_{\mathrm{WC}}}$ \\
\hline \multicolumn{7}{|c|}{$Z=0.004$} \\
\hline 120 & 300 & 0.8298 & 0.5105 & 0.0066 & 0.0089 & 0.3127 \\
\hline 60 & 300 & 0.3704 & 0.3126 & 0.0039 & 0.0113 & 0.0539 \\
\hline 60 & 500 & 0.8617 & 0.4617 & 0.1422 & 0.1089 & 0.2578 \\
\hline 40 & 300 & 0.3057 & 0.3057 & 0.0000 & 0.0000 & 0.0000 \\
\hline \multicolumn{7}{|c|}{$Z=0.008$} \\
\hline 120 & 300 & 1.1107 & 0.7354 & 0.0282 & 0.0167 & 0.3471 \\
\hline 60 & 300 & 0.4315 & 0.2113 & 0.0088 & 0.0186 & 0.2114 \\
\hline 40 & 300 & 0.4677 & 0.4168 & 0.0238 & 0.0372 & 0.0271 \\
\hline 30 & 300 & 0.3421 & 0.2971 & 0.0299 & 0.0528 & 0.0151 \\
\hline \multicolumn{7}{|c|}{$Z=0.040$} \\
\hline 120 & 0 & 1.1829 & 0.7646 & 0.1276 & 0.0034 & 0.2907 \\
\hline 120 & 300 & 1.8956 & 1.4398 & 0.2004 & 0.0048 & 0.2554 \\
\hline $120^{1}$ & 300 & 2.3190 & 1.7200 & 0.4339 & 0.0382 & 0.1651 \\
\hline 85 & 300 & 1.8955 & 1.4626 & 0.1641 & 0.0980 & 0.2688 \\
\hline $85^{1}$ & 300 & 2.2710 & 1.6190 & 0.3087 & 0.1723 & 0.3433 \\
\hline 60 & 0 & 0.5592 & 0.2083 & 0.0552 & 0.0009 & 0.2957 \\
\hline 60 & 300 & 1.8083 & 1.3294 & 0.2640 & 0.0083 & 0.2149 \\
\hline $60^{1}$ & 300 & 2.1382 & 1.5450 & 0.4515 & 0.0940 & 0.1417 \\
\hline 40 & 300 & 0.5960 & 0.2857 & 0.0537 & 0.0291 & 0.2566 \\
\hline $40^{1}$ & 300 & 0.5710 & 0.2221 & 0.0433 & 0.0231 & 0.3056 \\
\hline 25 & 300 & 0.4493 & 0.2091 & 0.0706 & 0.0602 & 0.1696 \\
\hline \multicolumn{7}{|c|}{ Predicted number ratios } \\
\hline$v_{\text {ini }}$ & $\frac{\mathrm{WR}}{\mathrm{O}}$ & $\frac{\mathrm{eWNL}}{\mathrm{WR}}$ & $\frac{\mathrm{eWNE}}{\mathrm{WR}}$ & $\frac{\mathrm{WN} / \mathrm{WC}}{\mathrm{WR}}$ & $\frac{\mathrm{WC}}{\mathrm{WR}}$ & $\frac{\mathrm{WC}}{\mathrm{WN}}^{2}$ \\
\hline \multicolumn{7}{|c|}{$Z=0.004$} \\
\hline 300 & 0.02 & 0.83 & 0.00 & 0.01 & 0.15 & 0.18 \\
\hline \multicolumn{7}{|c|}{$Z=0.008$} \\
\hline 300 & 0.05 & 0.74 & 0.05 & 0.07 & 0.22 & 0.28 \\
\hline \multicolumn{7}{|c|}{$Z=0.020^{3}$} \\
\hline 0 & 0.02 & 0.35 & 0.16 & 0.00 & 0.49 & 0.97 \\
\hline 300 & 0.07 & 0.66 & 0.05 & 0.04 & 0.25 & 0.35 \\
\hline \multicolumn{7}{|c|}{$Z=0.040$} \\
\hline 0 & 0.06 & 0.46 & 0.10 & 0.00 & 0.44 & 0.78 \\
\hline 300 & 0.16 & 0.63 & 0.12 & 0.05 & 0.24 & 0.32 \\
\hline $300^{1}$ & 0.18 & 0.59 & 0.15 & 0.06 & 0.26 & 0.36 \\
\hline
\end{tabular}

${ }^{1}$ Model computed with mass loss rates during the WR phase dependent on the metallicity (Crowther et al. 2002).

$2 \frac{\mathrm{WC}}{\mathrm{eWNL}+\mathrm{eWNE}}$.

3 Values obtained in Paper X.

WC phase is longer at higher metallicity as a result of the shift towards a lower value of the minimum initial mass of single stars needed to become a Wolf-Rayet star.

As already explained in Paper X, in the rotating stellar models a new phase of modest, but non-negligible duration, appears: the so-called transition WN/WC phase (see the values of $t_{\mathrm{WN} / \mathrm{WC}}$ given in Table 3 ). This phase is characterized by the simultaneous presence at the surface of both $\mathrm{H}$ - and He-burning products. The reason for this is the shallower chemical gradients which build up inside the rotating models. These shallower gradients inside the stars also produce a smoother evolution of the surface abundances as a function of time (see Fig. 14). As explained in Paper $\mathrm{X}$, for a transition WN/WC phase to 


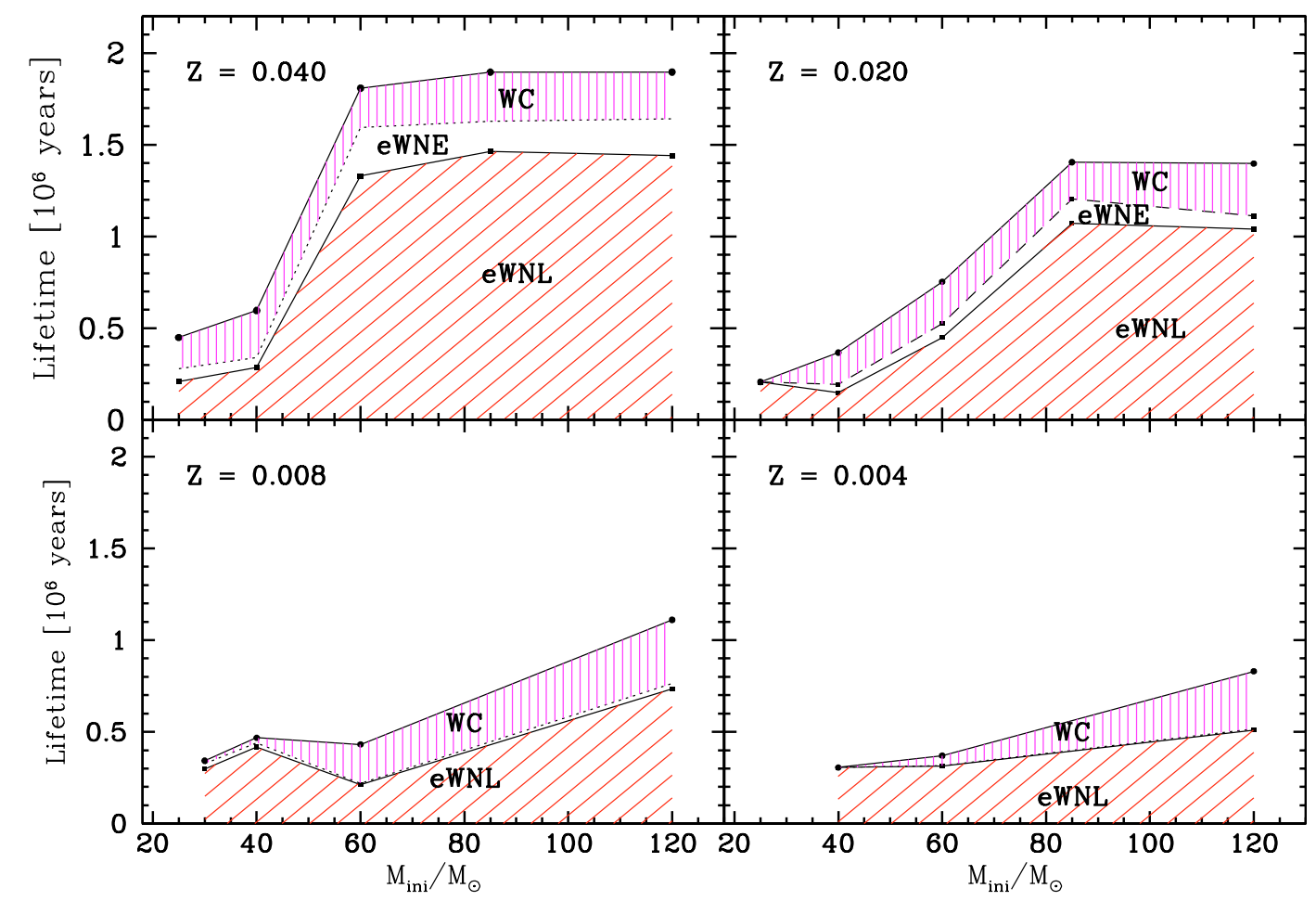

Fig. 9. Variation of the durations of the WR subphases as a function of the initial mass at various metallicities. All the models begin their evolution with $v_{\text {ini }}=300 \mathrm{~km} \mathrm{~s}^{-1}$.

occur, it is necessary to have - for a sufficiently long period - both an He-burning core and a CNO-enriched envelope. In general, in the highest mass stars, mass loss removes the CNO-enriched envelope too rapidly to allow a long transition WN/WC phase to occur. In the low mass range, the time spent in the WR phase is too short and the H-rich envelope too extended to allow He-burning products to diffuse up to the surface. Consequently, a significant transition WN/WC phase only appears in an intermediate mass range between $\sim 30$ and $60 M_{\odot}$ for $v_{\text {ini }}=300 \mathrm{~km} \mathrm{~s}^{-1}$. In general the duration of this phase also increases significantly when mass loss rates depending on the metallicity are used during the WR phase (see Sect 7).

\section{Comparison with the observed properties of Wolf-Rayet star populations}

Following Maeder (1991) and Maeder \& Meynet (1994), one can easily estimate the theoretical number ratio of WR to O-type stars in a region of constant star formation. This ratio is simply given by the ratio of the averaged lifetimes of a WR star to that of an OV-type star. The averaged lifetimes are weighted means of the lifetimes over the initial mass function (IMF). Assuming a Salpeter IMF slope $\left(\mathrm{d} N / \mathrm{d} M \propto M^{-(1+x)}\right.$, with $x=$ 1.35), considering the O-type and WR star lifetimes given in Tables 1 and 3 and the mass limits $M_{\mathrm{WR}}$ given in Table $2^{2}$, we obtain the predicted ratios given at the bottom of Table 3 .

\footnotetext{
${ }^{2}$ At $Z=0.004$, the value for the minimum initial mass of O-type stars is taken from the rotating models of Maeder \& Meynet (2001). At $Z=0.008$ we use the value obtained from the "enhanced" mass loss rate models of Meynet et al. (1994; see Table 2 in Maeder \& Meynet 1994).
}

Let us emphasize here that in the present work we assume that the $v_{\text {ini }}=300 \mathrm{~km} \mathrm{~s}^{-1}$ stellar models are well representative of the behaviour of the majority of the OB stars. In future studies, when information on the distribution of the initial rotation velocities will be available at different metallicities, it will certainly be interesting to convolve the theoretical results obtained for various initial velocities with these observed velocity distributions.

Looking at the results presented in Table 3, one can note that rotating models predict that

- The fraction of WR stars with respect to O-type stars increases with the metallicity.

- The fraction of eWNE with respect to WR stars increases with the metallicity, as does the fraction of WC stars (although the ratio seems to saturate around 0.25 for $Z \geq$ $0.020)$.

- The fraction of eWNL decreases with the metallicity again reaching a saturation level around 0.65 for $Z \geq 0.020$.

- The fraction of stars in the transition WN/WC phase has a non-monotonic behaviour, increasing from 0.01 at $Z=$ 0.004 to 0.07 at $Z=0.008$, and decreasing to values between $0.04-0.05$ for $Z \geq 0.020$.

Comparisons with observed number ratios are shown in Figs. 10 and 11. The values for the non-rotating models (both those computed with the present physics and those computed by Maeder \& Meynet 1994) are well below the observed values. The ratios predicted by the models with rotation are in much better agreement with the observations. In particular the ratios predicted by the rotating models are in quite good agreement with the observed ones at low metallicity. They give 


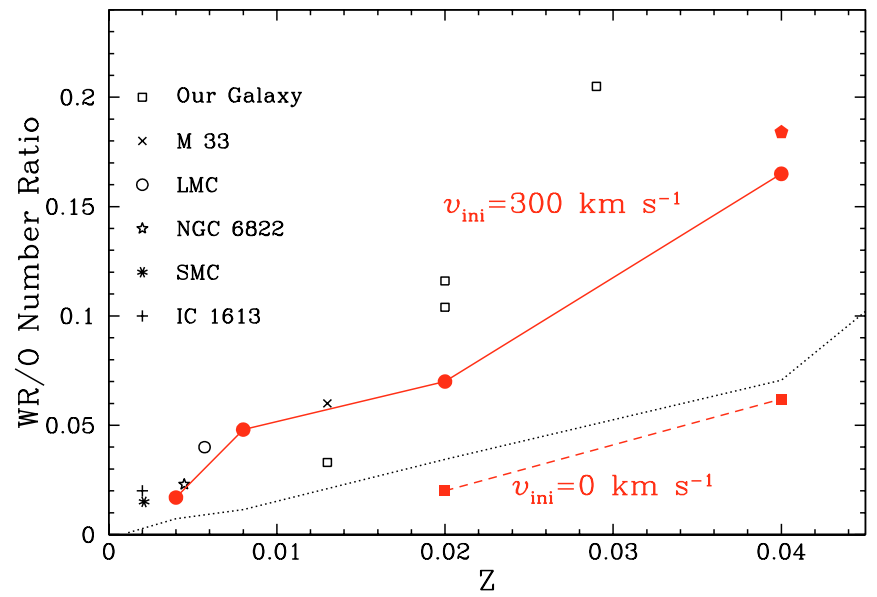

Fig. 10. Variation of the number ratios of Wolf-Rayet stars to O-type stars as a function of the metallicity. The observed points are taken from Maeder \& Meynet (1994). The dotted line shows the predictions of the models of Meynet et al. (1994) with normal mass loss rates. The continuous and the dashed lines show the predictions of the present rotating and non-rotating stellar models respectively. The black pentagon shows the ratio predicted by $Z=0.040$ models computed with the metallicity dependence of the mass loss rates during the WR phase proposed by Crowther et al. (2002).

predictions that are well in line with the results obtained by Foellmi et al. (2003a,b). Let us recall that these authors concluded that, in contrast to previous expectations, the binary channel for WR star formation does not seem to be a favoured scenario at low metallicity. Indeed, as already mentioned in the introduction, the fraction of binaries among the WR stars of the Small (40\%) and Large (30\%) Magellanic Cloud appears to be similar to that found in our Galaxy. Moreover, not all WR stars in a binary system must necessarily owe their existence to a Roche Lobe Overflow, since the two stars in a binary system may be sufficiently distant from each other to prevent that such a process occurs. Thus models from single massive stars should be able to reproduce more than half the number of observed WR stars in the Magellanic Clouds, a condition quite well fulfilled by the rotating models and not realized by the non-rotating ones. At high metallicity, the agreement is less good, although still much better than the one obtained from non-rotating models. Let us note that the samples at high metallicity do not have the same level of completeness as for the Magellanic Clouds. In the Clouds, the distance and metallicity are relatively well known quantities, moreover the internal extinction is relatively weak and thus does not constitute a severe barrier. The central regions of galaxies and of the Milky Way in particular do not offer such good conditions, and completeness problems are likely much more important than in the Magellanic Clouds.

In Fig. 11, the predicted values for the WC/WN number ratio are compared with observations in regions supposed to have undergone a constant star formation rate. The observations show that the WC/WN ratio increases with the metallicity along a relatively well defined relation. The observed point for the solar neighborhood is however well above the general

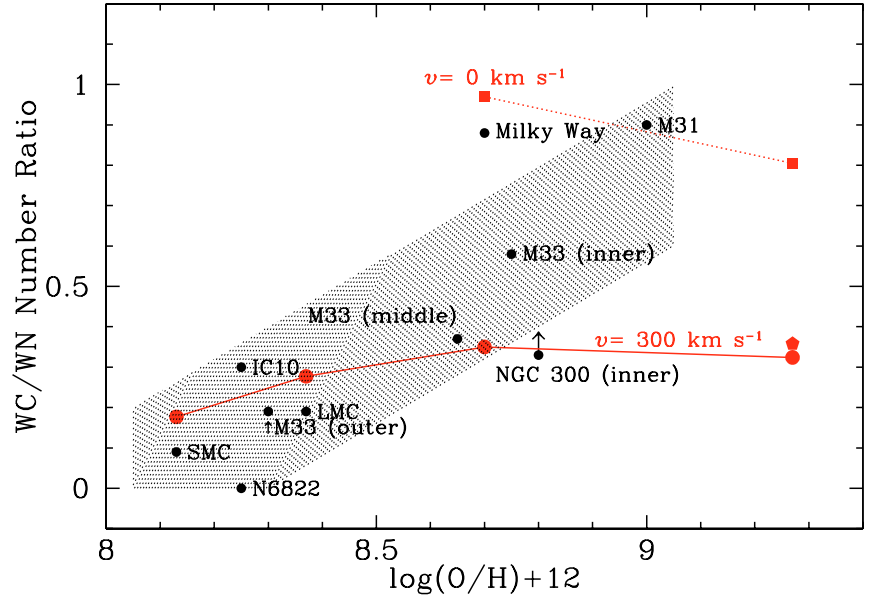

Fig. 11. Variation of the number ratios of $\mathrm{WN}$ to $\mathrm{WC}$ stars as a function of metallicity. The black circles are observed points taken from Massey \& Johnson (2001 and see references therein), except for the SMC (Massey \& Duffy 2001), for NGC 300 (Schild et al. 2002) and for IC10, for which we show the estimate from Massey \& Holmes (2002). The continuous and dotted lines show the predictions of the present rotating and non-rotating stellar models respectively. The black pentagon shows the ratio predicted by $Z=0.040$ models computed with the metallicity dependence of the mass loss rates during the WR phase proposed by Crowther et al. (2002).

trend. According to Massey (2003) this may result from an underestimate of the number of WN stars.

At low metallicity, models with rotation are well within the general observed trend. At solar metallicity they are just at the inferior limit and at twice the solar metallicity they are below the extrapolated observed trend. The non-rotating models predict (at least for $Z \geq 0.020$ ) significantly higher $\mathrm{WC} / \mathrm{WN}$ ratios. If the value predicted for the solar metallicity lies well above the real observed value at this metallicity (see Massey 2003), the one at twice the solar metallicity appears to be in good agreement with the extrapolated observed trend.

Taken at face value, it seems that rotating models overestimate the number of WN stars at high metallicity. Apart from invoking completeness problems in the observed sample, how might the comparisons between the ratios predicted by the rotating models and the observed ones be improved? We see two possible solutions: 1) From Fig. 11, it clearly appears that nonrotating stars, or more reasonably, stars rotating with relatively small velocities at high metallicity, would make the predicted points remain in the general observed trend. Slow rotators undergo little rotational mixing, thus they keep steep gradients of chemical composition in their interior. The fraction of the mass of the star presenting abundances characteristic of those of WN stars is therefore smaller than in a rotating star. This tends to decrease the WN lifetime. 2) The use of higher mass loss rates during the $\mathrm{WN}$ phase will tend to remove the H-rich envelope more rapidly, decreasing the WN lifetime and favouring an early entry into the WC phase.

The first solution is not reasonable for at least two reasons. Firstly, Fig. 10 shows that slowly rotating models are unable to account for the high fraction of WR stars observed at high metallicity. Secondly the observed number 


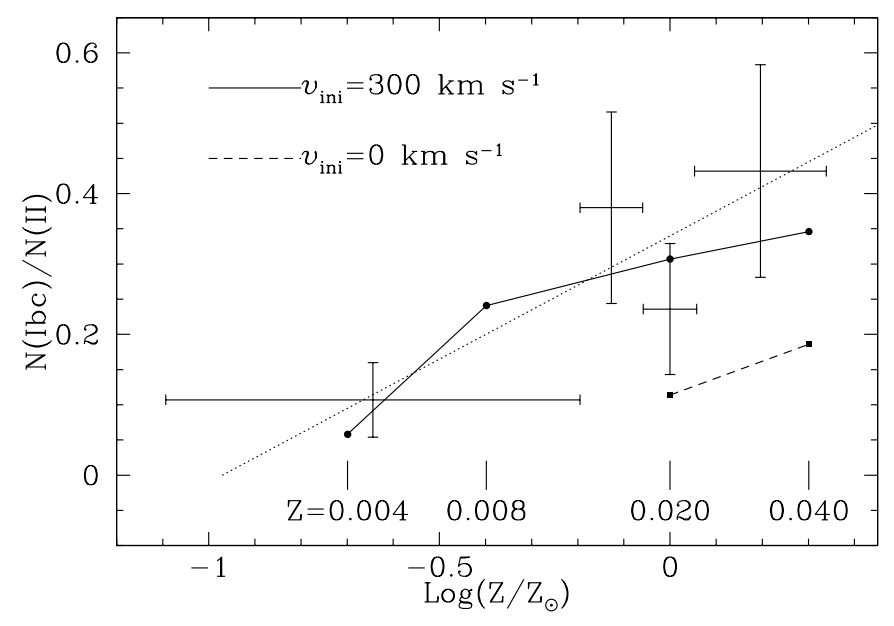

Fig. 12. Variation of the number ratios of type $\mathrm{Ib} / \mathrm{Ic}$ supernovae to type II supernovae. The crosses with the error bars correspond to the values deduced from observations by Prantzos \& Boissier (2003). The dotted line is an analytical fit proposed by these authors. The continuous and dashed line show the predictions of the present rotating and non-rotating stellar models.

ratio of type $\mathrm{Ib} / \mathrm{Ic}$ supernovae with respect to type II supernovae would also be severely underestimated by non-rotating models (Prantzos \& Boissier 2003, see also Sect. 6.1 below). The second solution, enhanced mass loss rates during the WN phase, appears more promising, although the effects of such an enhanced mass loss rate may be different according to the stage at which the star enters the WR phase. The most massive stars already enter the WN phase during the MS phase. An enhancement of the mass loss rates in that case can have the opposite effect of the one desired, namely it may lengthen the WN phase. Indeed a higher mass loss produces a more important reduction of the convective H-burning core, accompanied by a severe reduction of the central temperature. This tends to make the end of the H-burning phase longer, and thus to eventually increase the duration of the WN phase. Only an enhancement of the mass loss rates for those WN stars beyond the H-burning phase would reduce the WN phase.

Recently Crowther et al. (2002) proposed a metallicity dependence for the mass loss rates of WR stars comparable to that predicted for O-type stars, i.e. with $\left(Z / Z_{\odot}\right)^{1 / 2}$. We have recomputed the $Z=0.040$ models between 40 and $120 M_{\odot}$ with this new prescription. The lifetimes of the different WR subphases are given in Table 3 . We note that for the $120 M_{\odot}$ star, which enters the WR phase during the core H-burning phase, the stronger mass loss rates given by the new prescription lead to an increase of the WN phase and a decrease of the WC phase. The reason is just the one invoked above. In the case of the $40 M_{\odot}$ model, where only $17 \%$ of the WN phase is spent in the core H-burning phase, the metallicity dependent mass loss rates during the WR phase give a shorter WN phase and a slightly longer WC phase. It is likely that in the case of the $25 M_{\odot}$ model, which spends its whole WR phase in the post core $\mathrm{H}$-burning phase, the reduction of the WN phase and the enlargement of the WC phase would be more important. Supposing that for this $25 M_{\odot}$ stellar model the total WR lifetime is not much changed by the use of metallicity dependent mass loss rates during the WR phase, one can estimate the durations of the WN and WC phases one would obtain by simply assuming that the new $t_{\mathrm{WN}}$ is equal to that given in Table 3 divided by $(0.04 / 0.02)^{1 / 2}=1.4$ and the new $t_{\mathrm{WC}}$ is equal to $t_{\mathrm{WR}}-t_{\mathrm{WN}}$. If one does this one finds that the new $t_{\mathrm{WN}}$ and $t_{\mathrm{WC}}$ are 0.20 and $0.25 \mathrm{Myr}$ respectively. With these values, one obtains the predicted number ratios given in the last line of Table 3. One sees that the use of metallicity dependent mass loss rates during the WR phase does not much increase the predicted value for the WC/WN ratio at high metallicity (see also Fig. 11).

Thus in view of the above discussion, the two possibilities which presently appear as the most probable for reconciling the predicted $\mathrm{WC} / \mathrm{WN}$ ratio with the observed ones are 1) that the observed WC/WN ratio is overestimated as seems to be the case at least for the Milky Way (Massey 2003); 2) that the mass loss rates during the WN phase after the core H-burning phase are higher than presently assumed. One may also wonder what is the role played by binaries in this context.

\subsection{The type $I b / l c$ supernovae}

Current wisdom associates the supernovae of type $\mathrm{Ib} / \mathrm{Ic}$ with the explosion of WR stars, the H-rich envelope of which has been completely removed either by stellar winds and/or by mass transfer through Roche Lobe overflow in a close binary system. If we concentrate on the case of single star models, theory predicts that the fraction of type Ib/Ic supernovae with respect to type II supernovae should be higher at higher metallicity. The reason is the same as the one invoked to explain the increasing number ratio of WR to O-type stars with the metallicity $Z$, namely the growth of the mass loss rates with $Z$. Until recently very little observational evidence has been found confirming this predicted behaviour. The situation began to change with the work by Prantzos \& Boissier (2003). These authors have derived from published data the observed number ratios of type Ib/Ic supernovae to type II supernovae for different metallicities. The regions considered are regions of constant star formation rate. Their results are plotted in Fig. 12. Using the predicted ratios at solar metallicity deduced from our rotating models (Paper X), they concluded that rotating models were much better able than non-rotating ones to reproduce the observed ratio at solar metallicity.

We complement here the discussion of Prantzos \& Boissier (2003) by comparing theory to the observation at other metallicities. Theoretical ratios are simply given by the ratio $N_{\mathrm{Ib} / \mathrm{Ic}} / N_{\mathrm{II}}$, where $N_{\mathrm{Ib} / \mathrm{Ic}}$ is equal to the integration of the IMF over the range of $M_{\mathrm{WNE}}$ to $120 M_{\odot}$, where $M_{\mathrm{WNE}}$ is the minimum initial mass of the stars that end their lives as eWNE or WC stars, and where $N_{\text {II }}$ is the integration of the IMF between 8 and $M_{\mathrm{WNE}}$. To determine the values of $M_{\mathrm{WNE}}$ we used the same technique as the one used to determine $M_{\mathrm{WR}}$ and $M_{\mathrm{OWR}}$. We obtained 20.6, 22, 25 and 52 for $Z=0.040,0.020,0.008,0.004$ respectively. Looking at Fig. 12, it clearly appears that the conclusion of Prantzos \& Boissier (2003) is confirmed by the comparisons at low and high metallicities. Rotating models give a much better fit to the observed data than non-rotating models. This comparison can be viewed as a check of the lower initial 


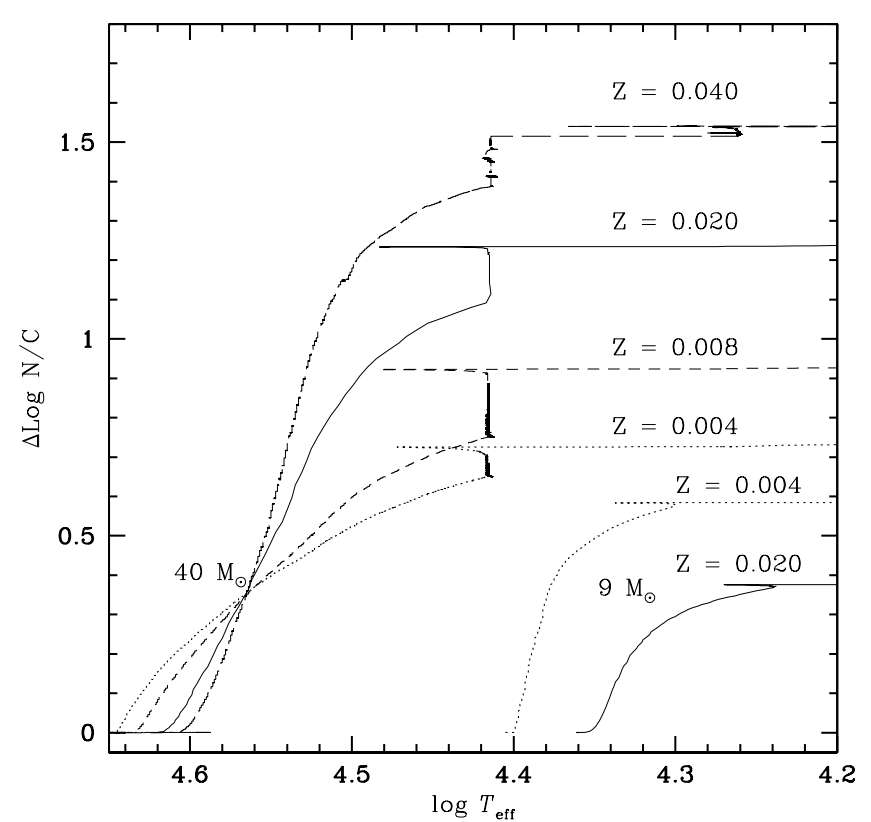

Fig. 13. Evolution during the MS phase of the N/C ratios (in number) at the surface of rotating stellar models as a function of the effective temperature. The differences in $\mathrm{N} / \mathrm{C}$ ratios are given with respect to the initial values.

mass limit $M_{\mathrm{WNE}}$ of the stars evolving into a WR phase without hydrogen, while the comparison between the observed and predicted number ratio of WR to O-type stars involves not only the value of the minimum initial mass of stars evolving into the WR phase but also the durations of the WR phase. In that respect the comparison with the supernovae ratios is a more direct check of the correctness of the value of $M_{\mathrm{WNE}}$ which at high metallicity appears rather close to $M_{\mathrm{WR}}$.

\section{Evolution of the surface abundances}

The effects of rotation on the surface abundance of massive stars at solar composition have been discussed by Heger \& Langer (2000), Meynet \& Maeder (2000, 2003). One of the main features is the enhancement of the N/C ratios at the surface during the MS phase. In Papers VII and VIII we obtained that, for a given initial mass and velocity, the surface enrichment induced by rotation is higher at lower metallicity. This is a consequence of the fact that at lower metallicity stars are more compact and have steeper internal gradients of $\Omega$ (see Papers VII and VIII). This implies strong shear mixing of the chemical elements and explains why in Fig. 13 the N/C ratio at the surface of the $9 M_{\odot}$ model at $Z=0.004$ is higher than for the corresponding model at $Z=0.020$.

For the more massive stars the situation is different. Indeed one can see in Fig. 13 that the N/C ratio at the end of the MS phase at the surface of the rotating $40 M_{\odot}$ at $Z=0.040$ is higher than the $\mathrm{N} / \mathrm{C}$ ratio obtained at the same stage at the surface of the $40 M_{\odot}$ at $Z=0.004$. Why is the behaviour of the most massive stars so different from that of the less massive ones? The reason is that, in the high mass star range, stellar winds become the dominant effect. High mass losses facilitate the surface enrichment by uncovering deep layers whose chemical composition has been changed by nuclear processing and/or rotational mixing.

Figure 14 shows the evolution of the surface abundances in rotating models with an initial mass of $40 M_{\odot}$. Before commenting on the effects of the metallicity, let us briefly recall the effects of rotation. In rotating models one can note the following differences with respect to the non-rotating ones:

- In rotating models the progressive changes of the abundances of $\mathrm{CNO}$ elements from the initial cosmic values to the values of the nuclear equilibrium of the $\mathrm{CNO}$ cycle are smoother. This is due to rotational mixing which smoothes internal chemical gradients.

- Due to rotational mixing, the change of abundances also occurs much earlier in the peeling-off process. This is also true for the changes of $\mathrm{H}$ and $\mathrm{He}$. We note that the nuclear equilibrium $\mathrm{CNO}$ values are essentially model independent as already stressed a long time ago (Smith \& Maeder 1991). This is true whether $\mathrm{H}$ is still present or not.

- In the rotating case, the transitions between the eWNE phase and the WC stage are smoother, so that there are some stars observed in the transition state which correspond to the so-called WN/WC stars (Conti \& Massey 1989; Crowther et al. 1995). These stars show simultaneously some ${ }^{12} \mathrm{C}$ and ${ }^{14} \mathrm{~N}$. They could also have some ${ }^{22} \mathrm{Ne}$ excess. Since the attribution of spectral types is a complex matter, it may even be that some of the stars in the transition stage are given a spectral type WNE or WC, thus we might well have a situation where a WN star would have some ${ }^{22} \mathrm{Ne}$ excess or a WC star would still have some ${ }^{14} \mathrm{~N}$ present.

- At the entry into the WC phase, the ${ }^{12} \mathrm{C}$ and ${ }^{16} \mathrm{O}$ abundances are lower in rotating models, and the abundance of $\mathrm{He}$ is higher.

- Also, the fraction of the WC phase spent with lower $\mathrm{C} / \mathrm{He}$ and $\mathrm{O} / \mathrm{He}$ ratios is longer in models with rotation.

- However, rotation does not affect the high level of the ${ }^{22} \mathrm{Ne}$ abundance during the WC phase. This is a consequence of the fact that most of this ${ }^{22} \mathrm{Ne}$ results from the transformation of the ${ }^{14} \mathrm{~N}$ produced by the $\mathrm{CNO}$ cycle in the previous $\mathrm{H}$-burning core. The value of the ${ }^{14} \mathrm{~N}$ and therefore that of the ${ }^{22} \mathrm{Ne}$ is fixed by the characteristics of the $\mathrm{CNO}$ at equilibrium, which in turn depends on the nuclear physics and not on the pecularities of the stellar models. It is interesting to mention here that the high overabundance of ${ }^{22} \mathrm{Ne}$ at the surface of the WC star predicted by the models is well confirmed by the observations (Willis 1999; Dessart et al. 2000).

Looking at Fig. 14, one can see how the evolution of the surface abundances is affected by rotation at four different metallicities. Among the most striking effects one can note:

- The eWNE phase is more extended at high metallicity than at low metallicity (see also Fig. 9). This is the result of the higher mass loss rates experienced at high metallicity. The removal of the outer layers by stellar winds is more rapid, therefore when the star enters the eWNE phase it does so at an earlier stage of the core He-burning phase, when the core 


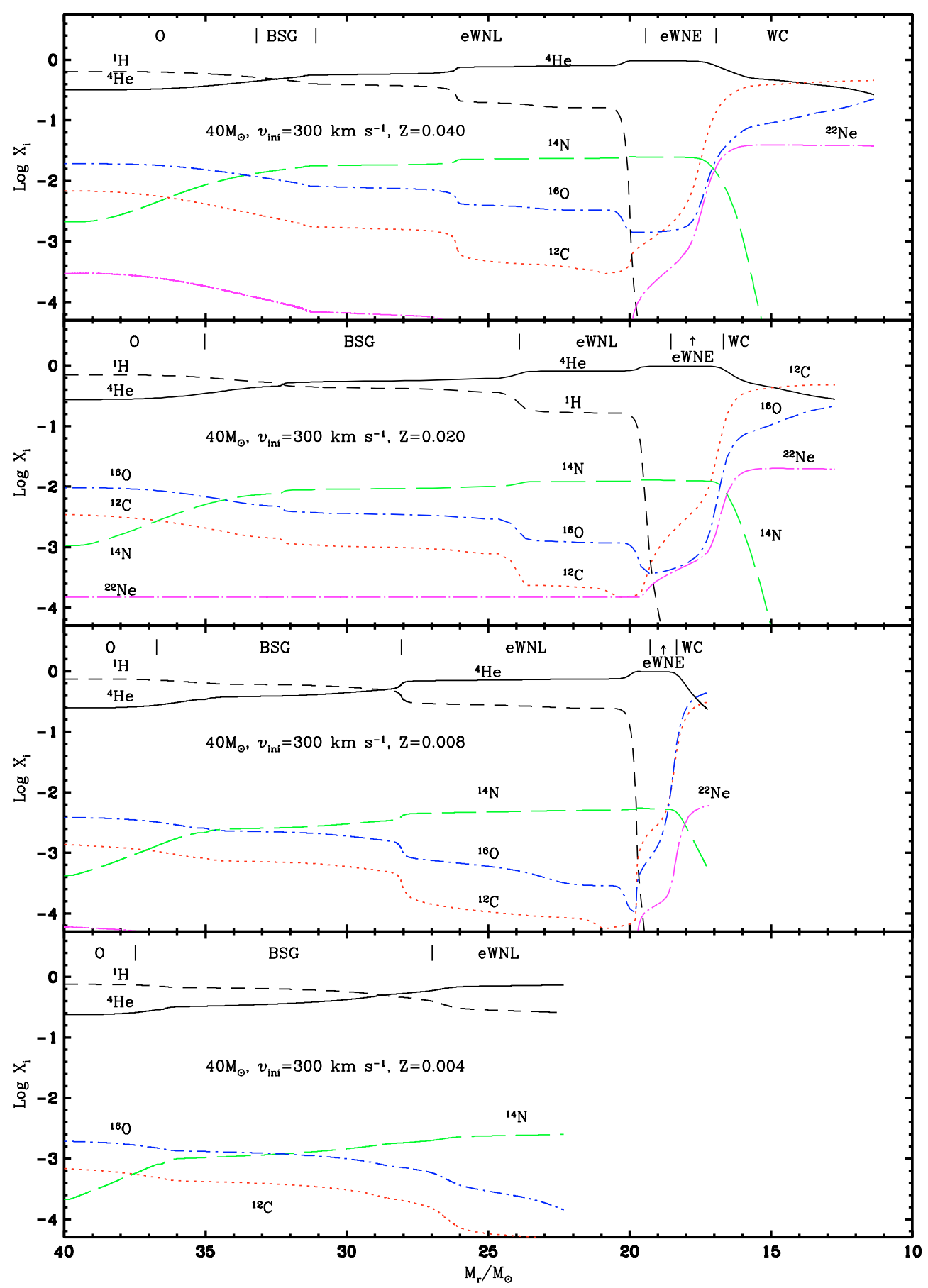

Fig. 14. Evolution as a function of the actual mass of the abundances (in mass fraction) of different elements at the surface of rotating $40 M_{\odot}$ stellar models at various metallicities.

is less massive. Thus the He-rich envelope extends over a larger fraction of the star.

- For the same physical reason as above, the star enters the WC phase at an earlier stage of the core He-burning phase at high metallicity. Therefore at high metallicity one expects that the surface abundances will be characterized by higher $\mathrm{He}$ abundance and larger $\mathrm{C} / \mathrm{O}$ ratios than at low metallicity. This has interesting consequences for the WC populations expected at various $Z$ (see below).

- The lower the metallicity, the less mass is lost by stellar winds and thus the less evolved is the stage at which the evolution ends. The $40 M_{\odot}$ stellar model at $Z=0.004$ never enters the WC phase. This model will explode as a core collapse type II supernova.

Figure 15 presents the evolution of the surface H-content as a function of the luminosity. The tracks go downwards in this diagram. Firstly there is an initial brightening without surface $\mathrm{H}$-depletion, then due to the concomitant effects of mixing and mass loss, the surface abundance of hydrogen decreases. The most striking effect of the metallicity is seen for the $120 \mathrm{M}_{\odot}$ stellar models. At higher metallicity, one notes that the high 


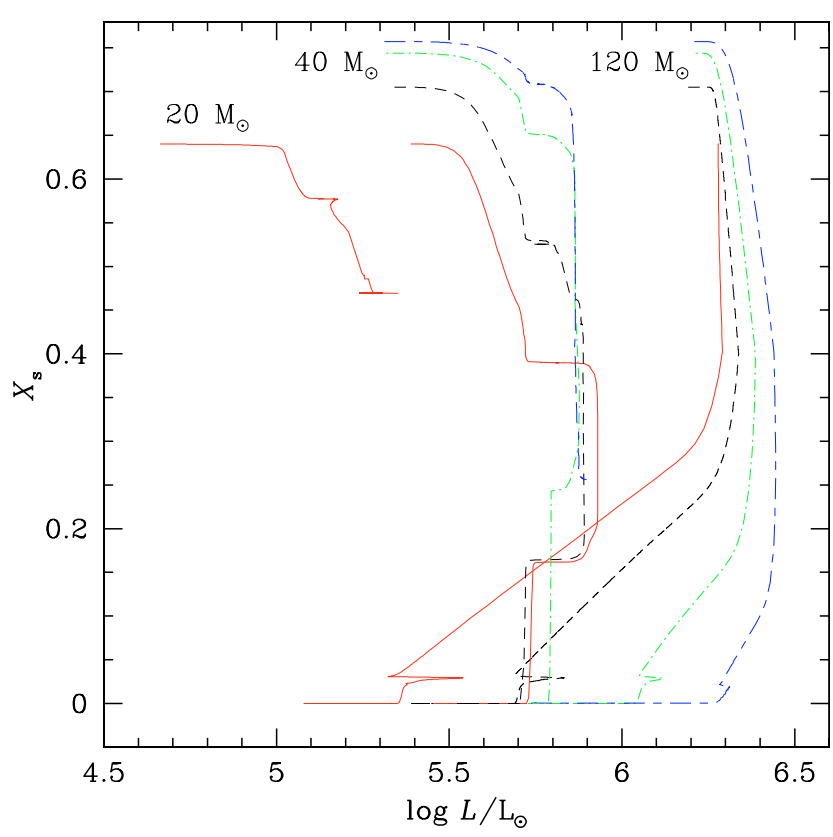

Fig. 15. Evolutionary tracks in the $X_{\mathrm{s}}$ versus $\log L / L_{\odot}$ plane, where $X_{\mathrm{s}}$ is the hydrogen mass fraction at the surface. The initial masses are indicated. Long-short dashed curves show the evolution of $Z=$ 0.004 models, dashed-dotted curves, short-dashed curves and continuous lines show the evolutions for $Z=0.008,0.020$ and 0.040 respectively.

mass loss prevents the track to go through the zone characterized by high luminosity and small surface hydrogen abundance. Only the models at low metallicity explore this domain of the $X_{\mathrm{S}}$ versus $\log L / L_{\odot}$ plane.

The evolution of the $(\mathrm{C}+\mathrm{O}) / \mathrm{He}$ ratio as a function of luminosity is shown in Fig. 16, which is the key diagram for WC stars as shown by Smith \& Maeder (1991). The track for a given mass goes up to the left. Indeed as a function of time, surface abundances characteristic of a more advanced He-burning stage appear at the surface resulting in an increase of the $(\mathrm{C}+\mathrm{O}) / \mathrm{He}$ ratio. At the same time mass loss reduces the actual mass of the star and therefore its luminosity. Since in rotating models there is a very progressive change of the surface abundance, all the models would seem to enter the WC phase with more or less the same $(\mathrm{C}+\mathrm{O}) / \mathrm{He}$ ratio. But this would be misleading since this transition phase is very short. Thus we decided here to plot the evolution of the surface ratios corresponding to the last $90 \%$ of the WC lifetime, so that the figure can give a better idea of the types of WC stars formed at the different metallicities.

One sees that at high metallicity, mainly late type WC stars are present, while at low metallicity, the late type WC stars are absent and earlier types are present. This is quite consistent with the observational fact that late type WC stars are only found in high metallicity regions (Smith \& Maeder 1991). Figure 16 also shows that a given WC subtype is reached at lower luminosity for a higher metallicity, a fact which is also consistent with the observed trend (Crowther et al. 2002). Such a behaviour results from the smaller mass at the entry in the WC phase for models at higher metallicity. The smaller mass

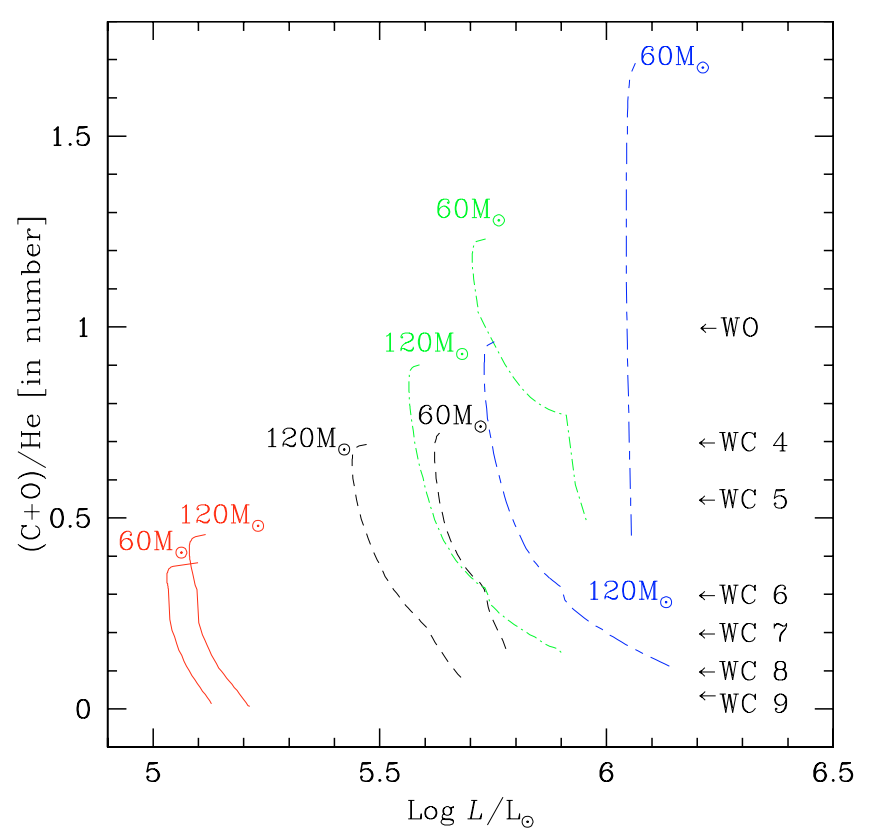

Fig. 16. Evolution of the ratios $(\mathrm{C}+\mathrm{O}) / \mathrm{He}$ as a function of the luminosity at the surface of 60 and $120 M_{\odot}$ rotating models for various initial metallicities (see text). Long-short dashed curves show the evolution of $Z=0.004$ models, dashed-dotted curves, short-dashed curves and continuous lines show the evolutions for $Z=0.008,0.020$ and 0.040 respectively. The correspondence between the $(\mathrm{C}+\mathrm{O}) / \mathrm{He}$ ratios and the different WC subtypes as given by Smith \& Maeder (1991) is indicated on the right of the figure.

in its turn is due mainly to the much longer WN phase experienced by the high metallicity stars, during which intense stellar winds peel off the star.

\section{Conclusion}

Rotation gives much better results than non-rotating models for the following observed features:

- the observed number ratio of WR to O-type stars for metallicities between 0.004 and 0.040 ;

- the observed ratio of WN to WC stars for metallicities lower than solar;

- the observed fraction of WR stars in the transition WN/WC phase;

- and the observed ratio of type Ib/Ic to type II supernovae at different metallicities.

Only in the case of the WC/WN ratio observed at high metallicity there may be a difficulty, although completeness problems may be part of the problem.

Interestingly, the features which were already well reproduced by non-rotating models, such as the abundance of ${ }^{22} \mathrm{Ne}$ at the surface of WC stars or the fact that late type WC stars are only observed at high metallicity, are also well accounted for by the present rotating models. This work further confirms that stellar rotation is an essential ingredient of massive star evolution, both for the MS phase and for the advanced stages like the WR stages and the Supernovae. We may therefore also 
anticipate that the nature of the remnants will be different depending on rotation and metallicity. In forthcoming papers we shall use the present rotating models to estimate the contribution of the WR stars to the synthesis of ${ }^{26} \mathrm{Al}$ in the Galaxy, and also to address the question of the rotation rates of pulsars and the progenitors of the collapsars suggested by Woosley (1993) to be possible progenitors of long gamma ray bursts.

\section{References}

Abbott, D. C., \& Conti, P. S. 1987, ARA\&A, 25, 113

Arnould, M., Meynet, G., \& Paulus, G. 1997, A\&A, 321, 452

Carr, J. S., Sellgren, K., \& Balachandran, S. C. 1999, ApJ, 530, 307

Chiosi, C., \& Maeder, A. 1986, ARA\&A, 24, 329

Conti, P. S. 1976, Mem. Soc. R. Sci. Liege, 9, 193

Conti, P. S., \& Massey, P. 1989, ApJ, 337, 251

Crowther, P. S., Smith, L. J., \& Willis, A. J. 1995, A\&A, 304, 269

Crowther, P. A., Dessart, L., Hillier, D. J., Abbott, J. B., \& Fullerton, A. W. 2002, A\&A, 392, 653

Cunha, K., Smith, V. V., Lambert, D. L., \& Hinkle, K. H. 2003, AJ, 126, 1305

Dessart, L., Crowther, P. A., Hillier, J. D., et al. 2000, MNRAS, 315, 407

Figer, D. F., Najarro, F., Gilmore, D., et al. 2002, ApJ, 581, 258

Fliegner, J., \& Langer, N. 1995, in IAU Symp, ed. K. A van der Hucht, \& P. M. Williams (Dordrecht: Kluwer), 163, 326

Foellmi, C., Moffat, A. F. J., \& Guerrero, M. A. 2003a, MNRAS, 338, 360

Foellmi, C., Moffat, A. F. J., \& Guerrero, M. A. 2003b, MNRAS, 338, 1025

Hamuy, M. 2003, in Core Collapse of Massive Stars, ed. C. L. Fryer (Dordrecht: Kluwer), in press [arXiv: astro-ph/0301006]

Heckman, T. M. 1999, in IAU Symp, ed. K. A. van der Hucht, G. Koenigsberger, \& P. R. J. Eenens, 193, 703

Heger, A., \& Langer, N. 2000, ApJ, 544, 1016

Heger, A., \& Woosley, S. E. 2004, in IAU Symp, 215, ed. A. Maeder, $\&$ P. Eenens, in press

Hirschi, R., Meynet, G., \& Maeder, A., A\&A, in press

Hjorth, J., Sollerman, J., Møller, P., et al. 2003, Nature, 423, 847

Iglesias, C. A., \& Rogers, F. J. 1996, ApJ, 464, 943

de Jager, C., Nieuwenhuijzen, H., \& van der Hucht, K. A. 1988, A\&AS, 72, 259

Kudritzki, R.-P., \& Puls, P. 2000, ARA\&A, 38, 613

Kunth, D., \& Sargent, W. L. W. 1981, A\&A, 101, L5

Langer, N. 1989, A\&A, 210, 93

Lípari, S., Terlevich, R., Díaz, R. J., et al. 2003, MNRAS, 340, 289

Maeder, A. 1987, A\&A, 178, 159

Maeder, A. 1991, A\&A, 242, 93
Maeder, A. 1992, A\&A, 264, 105

Maeder, A. 2002, A\&A, 392, 575

Maeder, A., Grebel, E. K., \& Mermilliod, J.-C. 1999, A\&A, 346, 459

Maeder, A., \& Meynet, G. 1994, A\&A, 287, 803

Maeder, A., \& Meynet, G. 2000a, A\&A, 361, 159 (Paper VI)

Maeder, A., \& Meynet, G. 2000b, ARA\&A, 38, 143

Maeder, A., \& Meynet, G. 2001, A\&A, 373, 555 (Paper VII)

Massey, P. 2003, ARA\&A, 41, 15

Massey, P., \& Johnson, O. 1998, ApJ, 505, 793

Massey, P., \& Duffy, A. S. 2001, ApJ, 550, 713

Massey, P., \& Holmes, S. 2002, ApJ, 580, L35

Meynet, G. 1999. in Variable and Non-spherical Stellar Winds in Luminous Hot Stars, ed. B. Wolf, O. Stahl, \& A. W. Fullerton, Lecture Notes in Physics, Springer, IAU Coll., 169, 377

Meynet, G. 2000. In Massive Stellar Clusters, ed. A. Lançon, \& C. M. Boily, ASP Conf. Ser., 211, 105

Meynet, G., \& Arnould, M. 2000, A\&A, 355, 176

Meynet, G., \& Maeder, A. 1997, A\&A, 321, 465 (Paper I)

Meynet, G., \& Maeder, A. 2000, A\&A, 361, 101 (Paper V)

Meynet, G., \& Maeder, A. 2003, A\&A, 404, 957 (Paper X)

Meynet, G., Arnould, M., Paulus, G., \& Maeder, A. 2001, Space Sci. Rev., 99, 73

Meynet, G., Maeder, A., Schaller, G., Schaerer, D., \& Charbonnel, C. 1994, A\&AS, 103, 97

Najarro, F. 2003, in CNO in the Universe, ed. C. Charbonnel, D. Schaerer, \& G. Meynet, ASP Conf. Ser., 304, 48

Nugis, T., \& Lamers, H. J. G. L. M. 2000, A\&A, 360, 227

Nugis, T., Crowther, P. A., \& Willis, A. J. 1998, A\&A, 333, 956

Prantzos, N., \& Boissier, S. 2003, A\&A, 406, 259

Prantzos, N., \& Diehl, R. 1996, Phys. Rep., 267, 1

Schaerer, D., \& Maeder, A. 1992, A\&A, 263, 129

Schaerer, D., Contini, T., \& Pindao, M. 1999, A\&AS, 136, 35

Schaller, G., Schaerer, D., Meynet, G., \& Maeder, A. 1992, A\&AS, 96, 269

Schild, H., Crowther, P. A., Abbott, J. B., \& Schmutz, W. 2002, A\&A, 397,859

Smith, L. F., \& Maeder, A. 1991, A\&A, 241, 77

Vink, J. S., de Koter, A., \& Lamers, H. J. G. L. M. 2000, A\&A, 362, 295

Vink, J. S., de Koter, A., \& Lamers, H. J. G. L. M. 2001, A\&A, 369, 574

Vuissoz, C., Meynet, G., Knödlseder, J., et al. 2004, New Astron. Rev., in press [arXiv: astro-ph/0311091]

Willis, A. J. 1999. In IAU Symp., 193, ed. K. A. van der Hucht, G. Koenigsberger, \& P. R. J. Eenens, ASP Conf Ser., 1

Woosley, S. E. 1993, ApJ, 405, 273

Woosley, S. E., \& Heger, A. 2004, in IAU Symp., 215, ed. A. Maeder, $\&$ P. Eenens, in press 